\title{
Substructure and galaxy formation in the Copernicus Complexio warm dark matter simulations
}

\author{
Sownak Bose ${ }^{1 \star}$, Wojciech A. Hellwing ${ }^{2,3}$, Carlos S. Frenk ${ }^{1}$, Adrian Jenkins ${ }^{1}$, \\ Mark R. Lovell ${ }^{4,5}$, John C. Helly ${ }^{1}$, Baojiu Li ${ }^{1}$, Violeta Gonzalez-Perez ${ }^{2}$, and Liang Gao ${ }^{1,6}$ \\ ${ }^{1}$ Institute for Computational Cosmology, Durham University, South Road, Durham, UK, DH1 3LE \\ ${ }^{2}$ Institute of Cosmology and Gravitation, University of Portsmouth, Portsmouth, UK, PO1 3FX \\ ${ }^{3}$ Janusz Gil Institute of Astronomy, University of Zielona Góra, ul. Szafrana 2, 65-516 Zielona Góra, Poland \\ ${ }^{4}$ GRAPPA Institute, Universiteit van Amsterdam, Science Park 904, 1098 XH Amsterdam, The Netherlands \\ ${ }^{5}$ Instituut-Lorentz for Theoretical Physics, Niels Bohrweg 2, NL-2333 CA Leiden, The Netherlands \\ ${ }^{6}$ National Astronomical Observatories, Chinese Academy of Sciences, 20A Datun Road, Chaoyang District, Beijing 100012, China
}

28 October 2016

\begin{abstract}
We use the Copernicus Complexio (COCO) high resolution $N$-body simulations to investigate differences in the properties of small-scale structures in the standard cold dark matter (CDM) model and in a model with a cutoff in the initial power spectrum of density fluctuations consistent with both a thermally produced warm dark matter (WDM) particle with a rest mass of $3.3 \mathrm{keV}$, or a sterile neutrino with mass $7 \mathrm{keV}$ and leptogenesis parameter $L_{6}=8.7$. The latter corresponds to the "coldest" model with this sterile neutrino mass compatible with the identification of the recently detected $3.5 \mathrm{keV}$ X-ray line as resulting from particle decay. CDM and WDM predict very different number densities of subhaloes with mass $\lesssim 10^{9} h^{-1} M_{\odot}$ although they predict similar, nearly universal, normalised subhalo radial density distributions. Haloes and subhaloes in both models have cuspy NFW profiles, but WDM subhaloes below the cutoff scale in the power spectrum (corresponding to maximum circular velocities $V_{\max }^{z=0} \leqslant 50 \mathrm{kms}^{-1}$ ) are less concentrated than their CDM counterparts. We make predictions for observable properties using the GALFORM semi-analytic model of galaxy formation. Both models predict Milky Way satellite luminosity functions consistent with observations, although the WDM model predicts fewer very faint satellites. This model, however, predicts slightly more UV bright galaxies at redshift $z>7$ than CDM, but both are consistent with observations. Gravitational lensing offers the best prospect of distinguishing between the models.
\end{abstract}

Key words: methods: numerical, $N$-body simulations - cosmology: dark matter - galaxies: evolution, high redshift

\section{INTRODUCTION}

Over the past three decades, advances in the scale and sophistication of numerical simulations have led to significant progress in studies of the non-linear phases of cosmological structure formation. Simulations have played a major role in establishing Lambda Cold Dark Matter ( $\Lambda \mathrm{CDM}$, hereafter just $\mathrm{CDM})$ as the standard model of cosmogony, helping link the theory to observations over a large range of scales and epochs, from temperature fluctuations in the cosmic microwave background (Planck Collaboration et al. 2014) through the large-scale distribution of galaxies (Colless et al. 2001; Zehavi et al. 2002; Hawkins et al. 2003; Tegmark et al. 2004;

^ E-mail: sownak.bose@durham.ac.uk
Cole et al. 2005; Eisenstein et al. 2005) to the inner structure of dark matter haloes (see Frenk \& White 2012, for a review).

Although it is almost certainly the case that the dark matter consists of non-baryonic elementary particles (Planck Collaboration et al. 2014), the identity of the particle remains a mystery. There are hotly debated claims that dark matter of different kinds has been indirectly detected. The "gamma-ray excess" observed towards the Galactic centre has been ascribed to annihilation radiation of cold dark matter (Hooper \& Goodenough 2011). On the other hand the $3.53 \mathrm{keV}$ X-ray line detected in the stacked spectrum of clusters (Bulbul et al. 2014) and, independently, in the Perseus cluster and Andromeda (Boyarsky et al. 2014) has been ascribed to the decay of a $7 \mathrm{keV}$ sterile neutrino (but see, e.g. Malyshev et al. 2014; Anderson et al. 2015; Riemer-Sørensen 2016 for different interpretations). These two kinds of dark matter would produce very 
similar large-scale structure but can give rise to large observable differences on small scales.

Warm dark matter (WDM) particles have non-negligible thermal velocities at early times which damp primordial density fluctuations below a free streaming scale. The position of the cutoff depends on the mass and the production mechanism of the warm particles. If they are thermally produced, the cutoff length scales inversely with the particle mass and, if the particle mass is in the $\mathrm{keV}$ range, the cutoff corresponds roughly to the scale of dwarf galaxies. Structure formation in such models has been extensively studied using simulations in the past few years (e.g. Colín et al. 2000; Bode et al. 2001; Viel et al. 2005; Knebe et al. 2008; Schneider et al. 2012; Lovell et al. 2012; Macciò et al. 2013; Lovell et al. 2014; Reed et al. 2015; Colín et al. 2015; Yang et al. 2015; Bose et al. 2016a; Horiuchi et al. 2016). The observed clumpiness of the Lyman- $\alpha$ forest sets a lower limit to the mass of a dominant thermally produced WDM particle of $m_{\mathrm{WDM}} \geqslant 3.3 \mathrm{keV}$ at $95 \%$ confidence (Viel et al. 2013); this is consistent with a lower limit set by the observed abundance of satellites in the Milky Way (Kennedy et al. 2014; Lovell et al. 2016). The lower limit to the mass of thermal WDM was increased to $m_{\mathrm{WDM}} \geqslant 4.35 \mathrm{keV}$ (95\% confidence) by Baur et al. (2016), who repeated the analysis of Viel et al. (2013) with an updated sample of QSO spectra from SDSS-III. These limits, however, depend on uncertain assumptions for thermal history for the intergalactic medium (Garzilli et al. 2015).

Warm dark matter in the form of sterile neutrinos can also form through resonant processes in the early universe (Shi \& Fuller 1999). In this case, the primordial fluctuation spectrum is more complicated and both the position and shape of the cutoff depend on the formation mechanism. In the "neutrino Minimal Standard Model" ( $\nu$ MSM, Asaka \& Shaposhnikov 2005; Boyarsky et al. 2009) (a simple extension of the Standard Model of particle physics) right-handed sterile neutrinos of $\mathrm{keV}$ mass $\left(M_{1}\right)$ make up a triplet alongside two other neutrinos of $\mathrm{GeV}$ mass $\left(M_{2}\right.$ and $\left.M_{3}\right)$. $M_{1}$ behaves as WDM. If $M_{2}$ and $M_{3}$ decay before the production of the dark matter, they can create a "lepton asymmetry" (i.e. an excess of leptons over antileptons). In the presence of this asymmetry, the production of the dark matter can be boosted by resonant production (Shi \& Fuller 1999). The result is a model with the correct abundance of dark matter that also explains active neutrino oscillations.

The leptogenesis parameter, $L_{6}$, which determines the asymmetry, affects the cutoff scale and shape of the power spectrum cutoff in a non-trivial way illustrated in Fig. 1. The CDM power spectrum is shown as a thick black line and sterile neutrino models with particle mass of $7 \mathrm{keV}$ and $L_{6}$ ranging from 4.6 to 525 are shown by colour lines. All these power spectra are essentially identical on scales larger than $\log \left[k /\left(h \mathrm{Mpc}^{-1}\right)\right] \sim 0.5$, but differ on smaller scales both in the shape and location of the cutoff which, furthermore, does not vary monotonically with $L_{6}$. The power spectrum of a thermal $3.3 \mathrm{keV}$ WDM model (the limiting mass consistent with the Lyman- $\alpha$ forest constraint of Viel et al. 2013) is also plotted. This has a similar cutoff scale as the $L_{6}=8.66$ model, which is the "coldest" possible sterile neutrino model with a $7 \mathrm{keV}$ particle mass.

In this paper we use the Copernicus Complexio (COCOWARM) high resolution $N$-body simulation to investigate the properties of subhaloes in a WDM model. This simulation was run prior to the discovery of the $3.5 \mathrm{keV}$ line and its initial power spectrum was chosen to correspond to a thermal $3.3 \mathrm{keV}$ WDM model. This turns out to have been a fortuitous choice since this power spectrum is very similar to that of the coldest $7 \mathrm{keV}$ sterile neutrino, so constraints on this model can be readily extended to all sterile neutrino models with a $7 \mathrm{keV}$ particle mass. The formation times, mass functions, spins, shapes, mass profiles and concentrations of haloes in this simulation were presented in Bose et al. (2016a). Here we focus on the properties of halo substructures.

The COCO simulations are amongst the highest resolution WDM $N$-body simulations of a cosmological volume performed to date (see Section 2. Previous simulations at higher mass resolution have focussed on properties of individual haloes (e.g. Lovell et al. 2014; Colín et al. 2015). Other WDM simulations of comparable mass resolution to ours (e.g. Schneider et al. 2013) followed smaller volumes. The advantage of the relatively high mass resolution and large volume of COCO is that it provides a large statistical sample of well-resolved dark matter haloes spanning nearly seven decades in mass. In particular, resolving the halo mass function down to $\sim 10^{7}-10^{8} h^{-1} M_{\odot}$, as COCO does, is a crucial input to studies that attempt to distinguish amongst different types of dark matter using strong gravitational lensing (Vegetti \& Koopmans 2009; Li et al. 2016).

Our simulations are numerically converged down to a halo peak circular velocity of $V_{\max }, \geqslant 10 \mathrm{kms}^{-1}$, thus allowing statistically meaningful studies of the satellites of the Milky Way. Furthermore, the high resolution of our simulations makes it possible to construct accurate merger trees of even such small haloes and, as a result, we can calculate their observable properties, using the Durham semi-analytical galaxy formation model, GALFORM (Cole et al. 2000; Lacey et al. 2016), a flexible and effective method to implement the best current understanding of galaxy formation physics into an $N$-body simulation.

The layout of this paper is as follows. In Section 2 we introduce the COCO simulation set, which includes both the WDM model of interest here and its CDM counterpart. In Section 3 we investigate the main properties of subhaloes: their population statistics, distribution and internal structure. In Section 4 we describe the GALFORM model and the modifications required for the WDM case, and compare to predictions for the CDM case. Finally, we summarise our results in Section 5.

\section{THE SIMULATIONS}

\subsection{Simulation details}

The $N$-body simulations used in this work, COCO, were introduced by Hellwing et al. (2016) and Bose et al. (2016a), as part of the Virgo Consortium programme. In short, COCO is a set of cosmological zoom-in simulations that follow about 12 billion high resolution dark matter particles, each of mass $m_{p}=1.135 \times$ $10^{5} h^{-1} M_{\odot}$. The resimulation region was extracted from the $\left(70.4 h^{-1} \mathrm{Mpc}\right)^{3}$ parent volume, Copernicus complexio Low Resolution (COLOR). COLOR and COCO assume cosmological parameters obtained from WMAP-7: $\Omega_{m}=0.272, \Omega_{\Lambda}=0.728, h=$ $0.704, n_{s}=0.967$ and $\sigma_{8}=0.81$. The simulations were performed using GADGET-3, an updated version of the publicly available GADGET-2 code (Springel et al. 2001a; Springel 2005; Springel et al. 2008). Substructures in the simulation were identified using the SUBFIND algorithm (Springel et al. 2001b). For a comprehensive description of the initial conditions and choice for the zoom-in region, we refer the reader to Hellwing et al. (2016) and Bose et al. (2016a).

COCO consists of two simulations with initial fluctuation power spectra corresponding to $\mathrm{CDM}$ (COCO-COLD) and to the 


\begin{tabular}{cccccccc}
\hline \hline Simulation & Cube length & $m_{\mathrm{WDM}}$ & $N_{p}$ & $V_{\mathrm{hr}}$ & $m_{p, \mathrm{hr}}$ & $N_{p, \mathrm{hr}}$ \\
& & & & & & \\
& & & & & & \\
$\mathrm{C}$ & & & & & \\
COLOR & $70.4 h^{-1} \mathrm{Mpc}$ & $3.3 \mathrm{keV}$ & $4,251,528,000$ & $70.4^{3} h^{-3} \mathrm{Mpc}^{3}$ & $6.196 \times 10^{6} h^{-1} M_{\odot}$ & $4,251,528,000$ & $1 h^{-1} \mathrm{kpc}$ \\
COCO & - & $3.3 \mathrm{keV}$ & $13,384,245,248$ & $\sim 2.2 \times 10^{4} h^{-3} \mathrm{Mpc}^{3}$ & $1.135 \times 10^{5} h^{-1} M_{\odot}$ & $12,876,807,168$ & $230 h^{-1} \mathrm{pc}$ \\
& & & & & \\
\hline \hline
\end{tabular}

Table 1. Simulation parameters assumed in COCO and the parent simulation, COLOR. Here, $m_{\mathrm{WDM}}$ is the mass of the thermal relic warm dark matter particle; $N_{p}$ is the total number of particles (of all types) used in the simulation; $V_{\mathrm{hr}}$ is the approximate volume of the high-resolution region at $z=0 ; m_{p}$, hr is the mass of an individual high-resolution dark matter particle; $N_{p, \mathrm{hr}}$ is the total number of particles of this species; and $\epsilon_{\mathrm{hr}}$ is the softening length. The parameter $m_{\mathrm{WDM}}$ is only relevant for COCO-WARM.

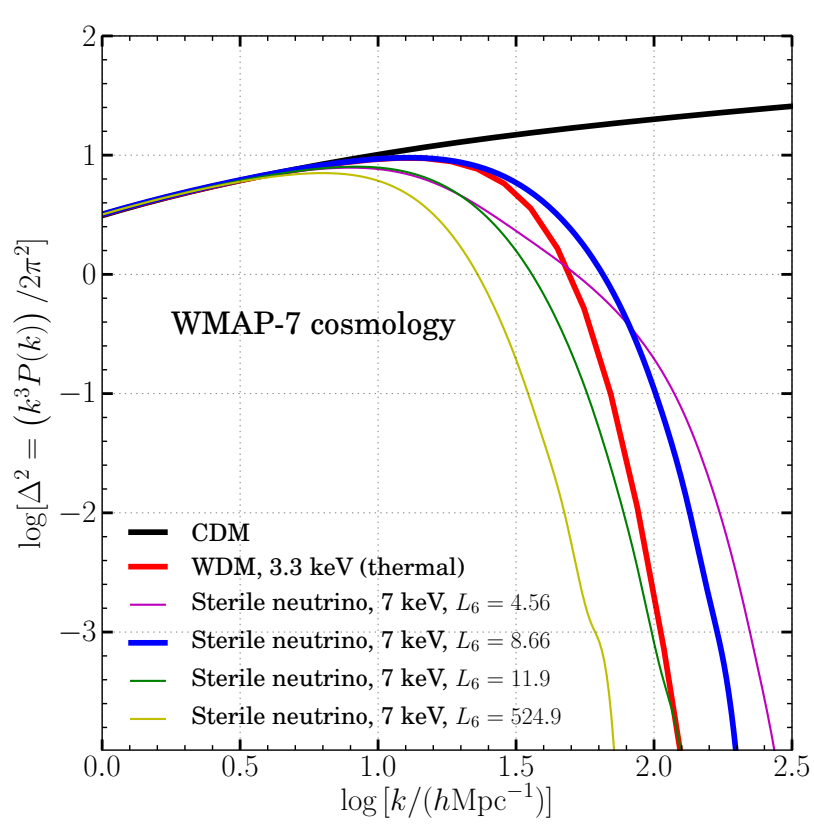

Figure 1. The (dimensionless) matter power spectrum for a thermal $3.3 \mathrm{keV}$ WDM (red, used in COCO-WARM), a sterile neutrino of mass $m_{\nu_{s}}=7 \mathrm{keV}$ and lepton asymmetry $L_{6}=8.66$ (blue) and CDM (black, used in COCOCOLD). Power is significantly suppressed at small scales for both thermal WDM and the sterile neutrino. The deviation from CDM occurs at almost identical scales: $\log \left[k\left(h / \mathrm{Mpc}^{-1}\right)\right] \gtrsim 1.0$. The excess of power at high- $k$ for $L_{6}=8.66$ compared to the $3.3 \mathrm{keV}$ case is negligible $(\lesssim 1 \%$ ) for the scales resolved in our simulations; see Appendix B in Lovell et al. (2016). The other thin coloured lines show power spectra for $7 \mathrm{keV}$ sterile neutrinos with different values of $L_{6}$, as labelled in the legend. Figure reproduced from Bose et al. (2016a).

$3.3 \mathrm{keV}$ thermal relic WDM (COCO-WARM); the initial fluctuation field had the same phases in both cases. COCO-WARM has a power spectrum with a sharp cutoff at small scales, which is approximated by the fitting formula:

$$
T(k)=\left(1+(\alpha k)^{2 \nu}\right)^{-5 / \nu},
$$

(Bode et al. 2001), where $T(k)$ is the transfer function relating the power spectra for CDM and WDM, $\nu=1.12$ is a constant, and $\alpha$ is determined by the mass of the particle:

$$
\alpha=0.049\left[\frac{m_{\mathrm{WDM}}}{\mathrm{keV}}\right]^{-1.11}\left[\frac{\Omega_{\mathrm{WDM}}}{0.25}\right]^{0.11}\left[\frac{h}{0.7}\right]^{1.22} h^{-1} \mathrm{Mpc} .
$$

(Viel et al. 2005). The CDM and WDM power spectra are then related by:

$$
P_{\mathrm{WDM}}(k)=T^{2}(k) P_{\mathrm{CDM}}(k) .
$$

The power spectra used in the COCO simulations are shown as thick lines in Fig. 1 and are discussed in Section 1. The main simulation parameters are listed in Table 1 . A projected density map of the COCO volume at $z=0$ is shown in Fig. 2 .

\subsection{Subhalo mass definitions}

The mass of a halo, $M_{\Delta}$, is defined as the mass within $r_{\Delta}$, the radius within which the average density is $\Delta$ times the critical density of the Universe at the redshift at which the halo is identified. Usually $\Delta=200$ is used to define the spherical overdensity within the virialised region of the halo, but we will also use $\Delta=50$ in order to compare with the results of the AQUARIUS project simulations (Springel et al. 2008). ${ }^{1}$ For the mass of a subhalo, $M_{\text {sub }}$, we take the mass identified by SUBFIND as the mass that is gravitationally bound to the subhalo.

\subsection{Identification and removal of numerical artefacts}

Since our analysis is primarily concerned with the properties of dark matter subhaloes and the galaxies that form in them, it is important to ensure that the merger trees have been pruned of the artificial structures that form from evolution from a power spectrum with a resolved cutoff. Spurious structures along filaments were apparent in the first WDM simulations (Bode et al. 2001) but were only subsequently recognised as an effect of particle discreteness by Wang \& White (2007). A technique to eliminate spurious objects in post-processing was developed by (Lovell et al. 2014), while Angulo et al. (2013) and Hobbs et al. (2016) have proposed modifications to the $N$-body simulation method itself to prevent the formation of such objects in the first place.

Wang \& White (2007) found that a large fraction of the spurious haloes can be removed by rejecting objects with mass below a

1 Note that in the Springel et al. (2008), $r_{50}$ is the radius as which the mean density is $200 \Omega_{m}$. In COCO, this would correspond to roughly $r_{54}$. 

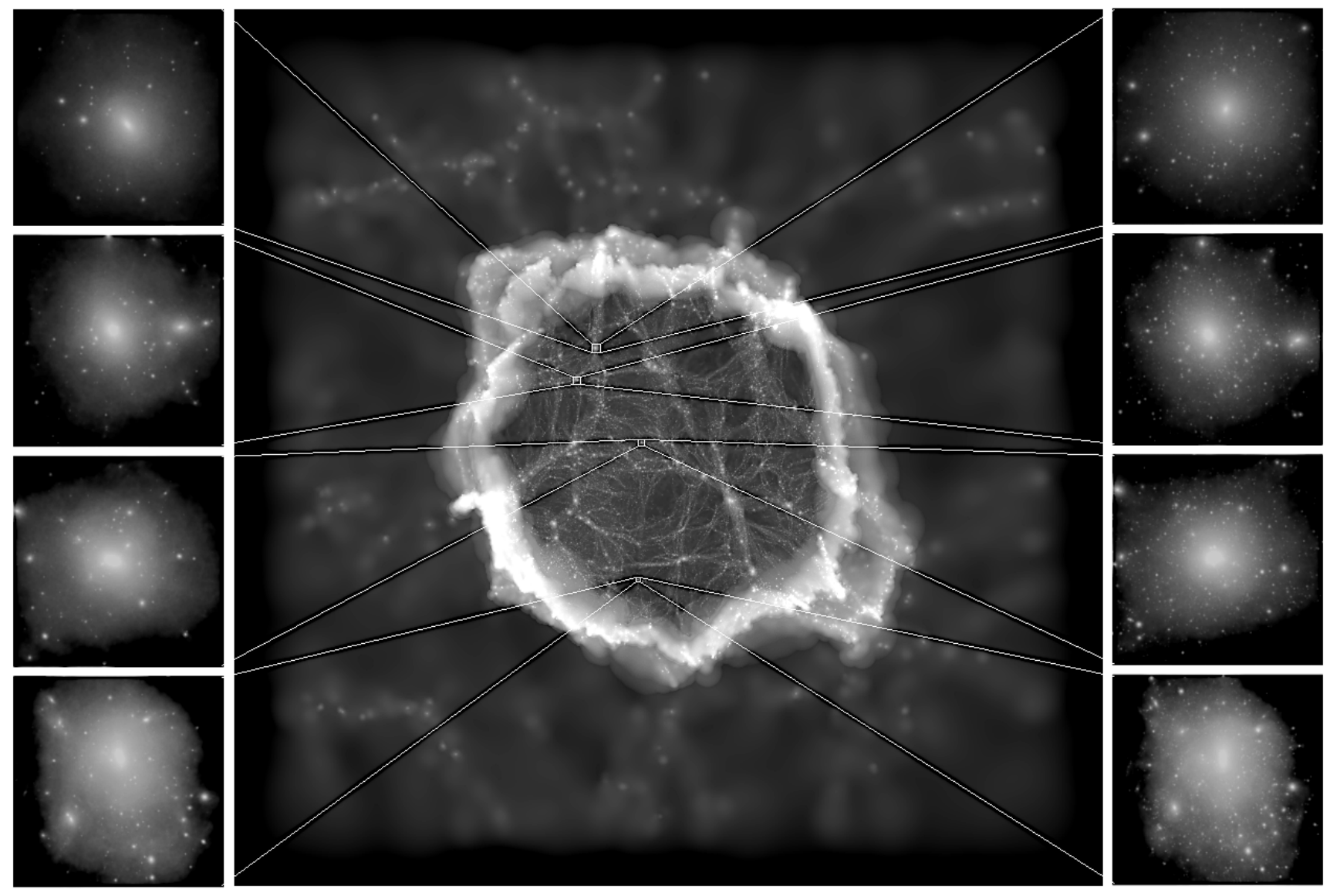

Figure 2. Projected density map in a slice of dimensions $(70.4 \times 70.4 \times 1.5) h^{-1} \mathrm{Mpc}$ centred on the coco high resolution region at $z=0$. The intensity of the image scales with the number density of particles in the region. The side panels show zooms of a sample of haloes identified at $z=0$, matched between COCO-WARM (left) and COCO-COLD (right)

resolution-dependent threshold:

$$
M_{\lim }=10.1 \bar{\rho} d k_{\text {peak }}^{-2}
$$

where $\bar{\rho}$ is the mean matter density in the universe, $d$ is the mean interparticle separation and $k_{\text {peak }}$ is the spatial frequency at which the dimensionless input power spectrum, $\Delta^{2}(k)$, has its maximum. Lovell et al. (2014) found that particles in spurious haloes in WDM originate from Lagrangian patches that are much flatter than the corresponding Lagrangian patches in CDM.

Lovell et al. (2014) devised the following procedure for identifying spurious haloes. Defining $M_{\max }$ as the maximum mass attained by a halo during its evolution, and $s_{\text {half }- \text { max }}$ as the sphericity ( $c / a$, where $c$ is the minor and $a$ the major axis of a uniform density ellipsoid with the same inertia tensor) of the halo particles (at high redshift) when it attains half of its maximum mass, we: (1) discard all (sub)haloes with $s_{\text {half }-\max }<s_{\text {cut }}$, irrespective of mass, and (2) for those that pass (1), remove (sub)haloes with $M_{\max }<0.5 M_{\text {lim }}$. The threshold sphericity in step (1) is chosen such that $99 \%$ of CDM haloes are rounder than $s_{\text {cut }}$. This threshold sphericity needs to be calculated for haloes identified at each redshift; at $z=0, s_{\text {cut }}=0.165$. The factor of 0.5 in step (2) was obtained by comparing different resolution levels of warm dark versions of the AQUARIUS simulations (see Lovell et al. 2014, for details). We apply this procedure to (sub)haloes in every snapshot in our simulation; branches in the merger tree that contain a spurious object are pruned from the tree.

\section{DARK MATTER SUBSTRUCTURE}

In this section we study the dark matter substructure in the COCOCOLD and COCO-WARM simulations, quantifying their abundance, distribution and internal structure. The general trend we find is that the largest subhaloes in COCO-WARM and COCO-COLD are indistinguishable but differences become increasingly significant below $\sim 5 \times 10^{9} h^{-1} M_{\odot}$.

\subsection{The abundance of subhaloes}

Fig. 3 shows the present-day differential mass function of subhaloes, $\mathrm{dn} / \mathrm{d} \log \left(\mathrm{M}_{\mathrm{sub}}\right)$, as a function of mass, $M_{\mathrm{sub}}$, in COCOCOLD (blue) and COCO-WARM before (green) and after (red) the removal of artefacts. The lower panel shows the ratio of abundances in COCO-WARM relative to COCO-COLD. The mass function, $\mathrm{dn} / \mathrm{d} \log \left(\mathrm{M}_{\mathrm{sub}}\right)$, is normalised by noting that the irregular volume of the high resolution region has a mean density roughly equal to the mean matter density in the Universe. Combining this with the total mass represented by high resolution particles, we can estimate the volume of the high resolution region. 


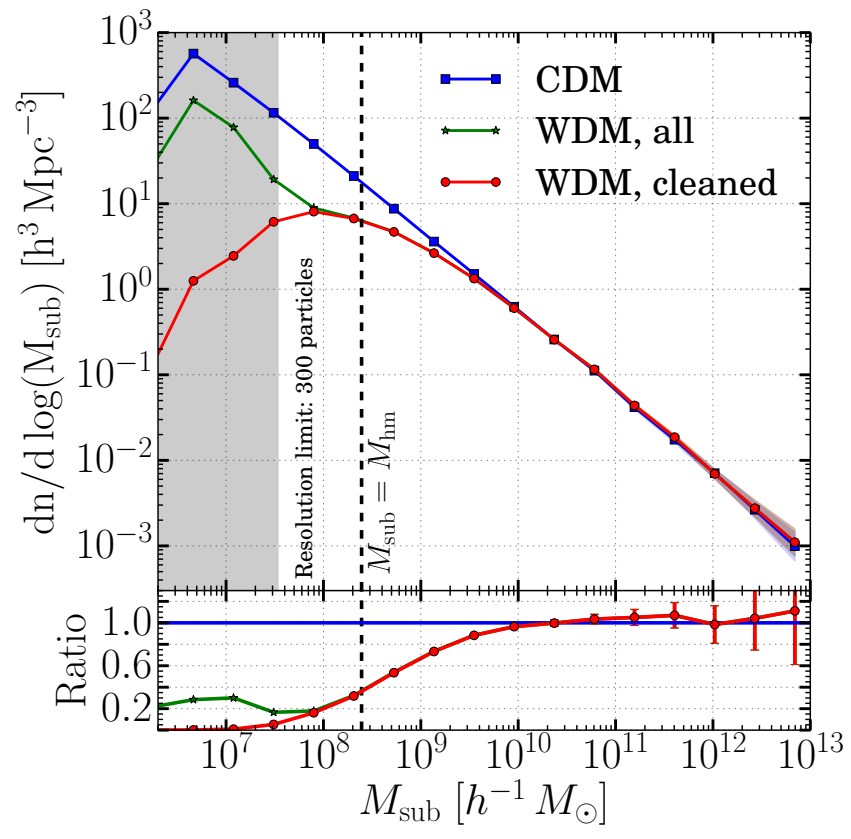

Figure 3. Upper panel: the number density of subhaloes as a function of subhalo mass, $M_{\mathrm{sub}}$, for COCO-COLD (blue), COCO-WARM with all objects included (green), and COCO-WARM with spurious structures removed (red). The shaded region around each curve represents the Poisson uncertainty in the number counts in that bin. The vertical black dashed line marks the half-mode mass, $M_{\mathrm{hm}}$, for the $3.3 \mathrm{keV}$ thermal relic. The grey shaded region demarcates the resolution limit of our simulations, set at 300 particles, which was determined by requiring convergence of the mass function compared with the lower-resolution version of COCO-COLD, COLOR-COLD. Lower panel: the ratio of the two COCO-WARM mass functions to the COCOCOLD mass function.

For $M_{\text {sub }}>10^{10} h^{-1} M_{\odot}$ the three mass functions agree very well. These haloes have masses well above the free streaming scale and no spurious objects form on these scales. Below $M_{\text {sub }} \sim$ $5 \times 10^{9} h^{-1} M_{\odot}$, the COCO-WARM mass function begins to peel off from COCO-COLD and by $\sim 3 \times 10^{8} h^{-1} M_{\odot}$ it is suppressed by a factor of two. This mass is close to the "half-mode mass", $M_{\mathrm{hm}}$, defined as the mass associated with the wavenumber, $k_{\mathrm{hm}}$, at which the transfer function in Eq. 1 falls to half the CDM value:

$$
k_{\mathrm{hm}}=\frac{1}{\alpha}\left(2^{\nu / 5}-1\right)^{1 / 2 \nu}=\frac{2 \pi}{\lambda_{\mathrm{hm}}} \approx 34 h \mathrm{Mpc}^{-1} \text {. }
$$

The half-mode mass (linearly extrapolated to $z=0$ ) is then:

$$
M_{\mathrm{hm}}=\frac{4 \pi}{3} \bar{\rho}\left(\frac{\lambda_{\mathrm{hm}}}{2}\right)^{3} \approx 2.5 \times 10^{8} h^{-1} M_{\odot} .
$$

Fig. 3 shows that the abundance of subhaloes in COCO-WARM is suppressed by a factor of three at $M_{\mathrm{hm}}$. Spurious subhaloes begin to dominate the mass function at a mass an order of magnitude below $M_{\mathrm{hm}}$. Before that happens, and still well above the resolution limit, at $M_{\text {sub }} \sim 10^{8} h^{-1} M_{\odot}$, the "cleaned" COCO-WARM mass function (i.e. after subtraction of spurious objects) is already a factor of 5 below the CDM case and shows a sharp turnover. The lower panel in the figure shows these trends more clearly. Removal of the spurious subhaloes is clearly important to obtain an accurate prediction for the abundance of low-mass galaxies in WDM models.

The statistics in COCO are good enough to allow the subhalo mass function to be calculated for different parent (host) halo

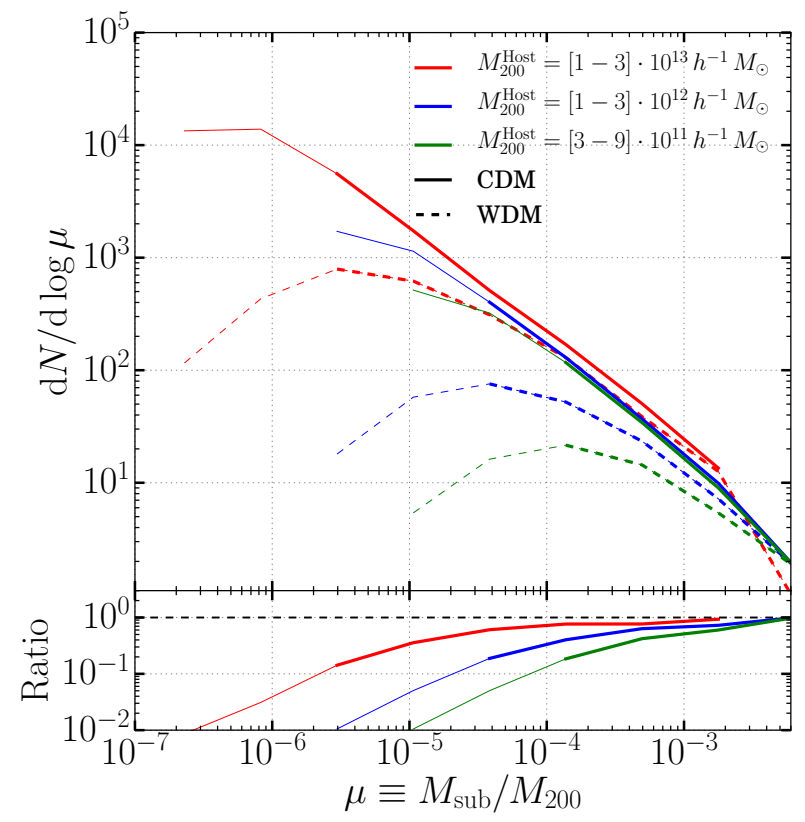

Figure 4. Upper panel: the stacked differential subhalo mass function as a function parent halo mass, expressed in units of $M_{\mathrm{sub}} / M_{200}$. The CDM case is shown with solid lines and the WDM case with dashed lines. The different colours correspond to different host halo mass ranges as indicated in the legend. The lines become thinner when a given subhalo has fewer than 300 particles i.e., when $\mu \times M_{200 \text {, mid }}^{\text {host }}>300 m_{p}$, where $M_{200 \text {, mid }}^{\text {host }}$ is the centre of the host halo mass bin, and $m_{p}$ is the high resolution particle mass. Lower panel: ratio of the differential subhalo mass functions in WDM to those in CDM.

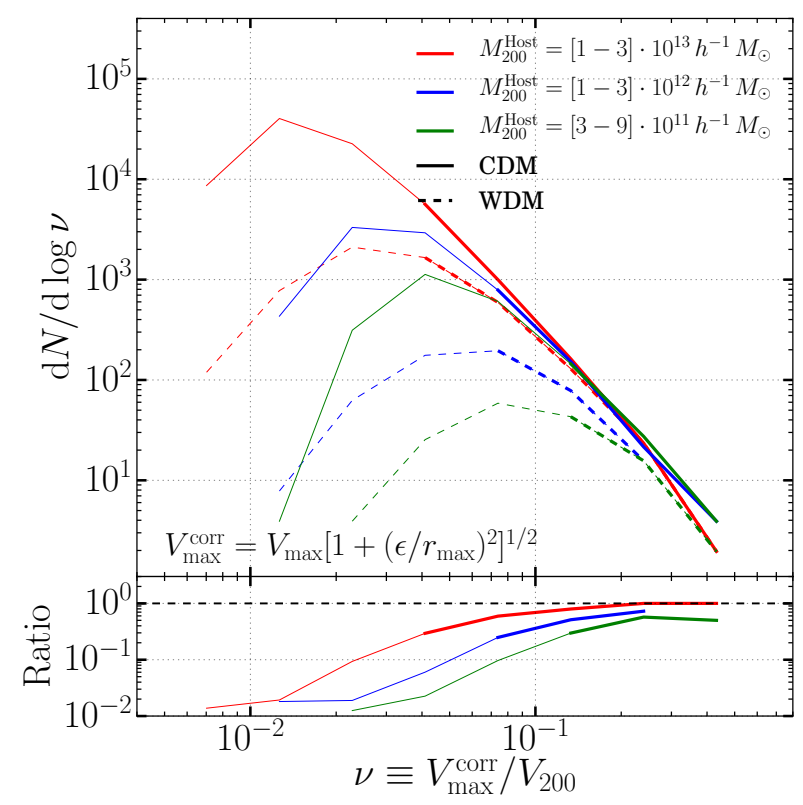

Figure 5. As Fig. 4, but with subhalo abundance expressed as a function of $V_{\max }^{\text {corr }} / V_{200}$, where $V_{\max }^{\text {corr }}$ is the maximum circular velocity, $V_{\max }$, corrected for the effects of gravitational softening as indicated in the legend (see main text). The lines become thinner when $V_{\max }<10 \mathrm{kms}^{-1}$, which is the circular velocity to which the simulations are complete. 


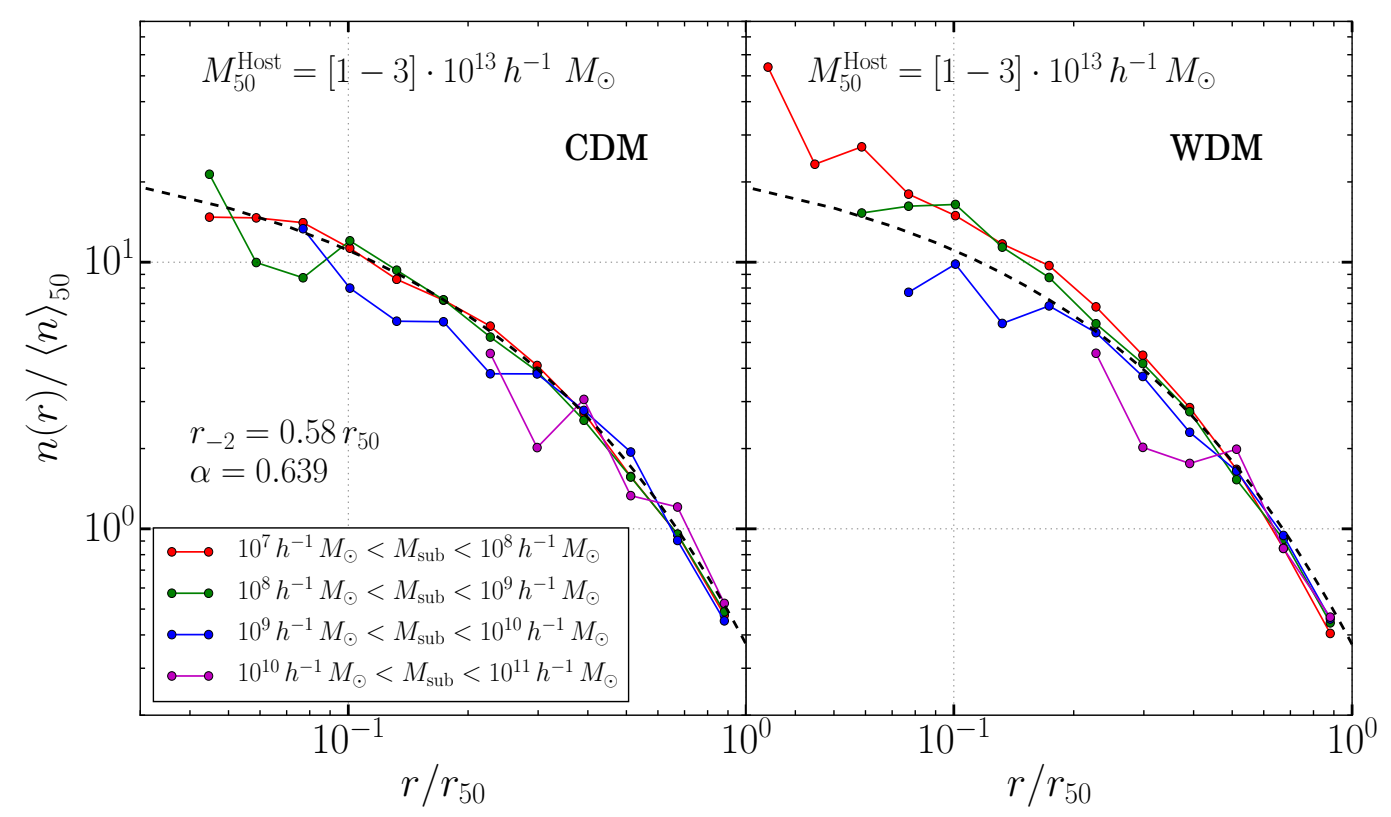

Figure 6. Stacked radial number density profiles of subhaloes, $n(r)$, in different mass ranges (different colours), normalised to the mean number density in that mass range within $r_{50}\left(\langle n\rangle_{50}\right)$. The profiles are plotted as a function of the distance from the host halo centre (with mass $M_{50}^{\text {Host }}=[1-3] \cdot 10^{13} h^{-1} M_{\odot}$ ). Left: CDM; right: WDM. The dashed black line shows the Einasto profile fit to the COCO-COLD profiles, with the fit parameters $r_{-2}$ and $\alpha$ quoted in the plot. Only subhaloes with more than 300 particles are shown.

masses. The result is shown in Fig. 4, which gives the (stacked) differential mass functions of subhaloes as a function of the relative mass, $\mu \equiv M_{\mathrm{sub}} / M_{200}$ (i.e., the subhalo mass in units of the parent halo mass), in three bins of host halo mass. The COCO-COLD functions are shown with solid lines and the COCO-WARM ones with dashed lines. In both cases, the lines become thinner for subhaloes with fewer than 300 particles. The lower panel of Fig. 4 shows the ratio of the differential subhalo mass functions in COCO-WARM to those in COCO-COLD.

The solid lines in the upper panel of Fig. 4 illustrate the invariance of the CDM subhalo mass function, when expressed in terms of $\mu$, previously seen by Springel et al. (2008), Gao et al. (2012) and Cautun et al. (2014). The relation is well described by a nearly universal power law (the turnover in the mass function towards low masses is due to incompleteness caused by the resolution of the simulations.) The scale invariance is broken in the case of COCOWARM, where the mass function deviates from a power law at larger values of $\mu$ for smaller host haloes. This can be understood from the fact that, when expressed in units of the host halo mass, the cutoff scale (or, equivalently, $M_{\mathrm{hm}}$ ) is reached earlier in lower host masses. The abundance of subhaloes is only slightly affected for a host of mass $M_{200}=10^{13} h^{-1} M_{\odot}$, but is strongly suppressed for $M_{200}=10^{11} h^{-1} M_{\odot}$ (for which $\mu=10^{-3}$ corresponds to $\left.M_{\text {sub }}=10^{8} h^{-1} M_{\odot}\right)$.

Given the ambiguity in the definition of subhalo mass, an alternative property used to count bound substructures is in terms its value of $V_{\max }$, defined as the maximum of the circular velocity curve. Furthermore, this quantity is measurable for many real satellites (where the rotation curve of the satellite can be measured) so it provides a better way than the mass to compare the simulations to observations. The upper panel of Fig. 5 shows the " $V_{\max }$ function," that is the number of subhaloes as a function of $\nu \equiv V_{\max } / V_{200}$, where $V_{200}$ is the circular velocity of the parent halo at $r_{200}$. Springel et al. (2008) found that the convergence of the $V_{\max }$ function improves markedly when $V_{\max }$ is corrected for the effects of gravitational softening:

$$
V_{\max }^{\mathrm{corr}}=V_{\max }\left[1+\left(\epsilon / r_{\max }\right)^{2}\right]^{1 / 2} .
$$

This correction is important for subhaloes whose $r_{\max }$ (the radius at which $V_{\max }$ occurs) is not much larger than the gravitational softening, $\epsilon$. The gravitational softening adopted in $\mathrm{COCO}$ $\left(\epsilon=230 h^{-1} \mathrm{pc}\right)$ is quite small and we have checked that the correction does not have a significant impact in our results. For CDM, the scale invariance of the subhalo abundance expressed in terms $V_{\max }$ is much clearer than when the abundance is expressed in terms of mass, as may be seen by comparing Figs. 4 and 5, confirming the earlier results of Moore et al. (1999); Kravtsov et al. (2004); Zheng et al. (2005); Springel et al. (2008); Weinberg et al. (2008); Klypin et al. (2011); Wang et al. (2012); Cautun et al. (2014)

It is clear from Figs. 4 and 5 that, when expressed in dimensionless units such as $\mu$ or $\nu$, the subhalo abundance in CDM is close to universal, independent of parent halo mass. In WDM the cutoff in the power spectrum breaks this approximately self-similar behaviour and the subhalo abundance is no longer a universal function.

\subsection{Radial distribution}

Perhaps surprisingly, Springel et al. (2008) found that the normalised radial number density distribution of subhaloes is approximately independent of subhalo mass (see also Ludlow et al. 2009; Hellwing et al. 2016). Han et al. (2016) has provided a simple analytical model that explains this feature, as well as the shape of the subhalo mass function in CDM, as resulting from tidal stripping. The subhalo radial distributions in COCO are shown in Fig. 6, which gives the radial number density of subhaloes in different mass ranges, normalised by the mean number density of subhaloes within $r_{50}$ at $z=0$. The distribu- 


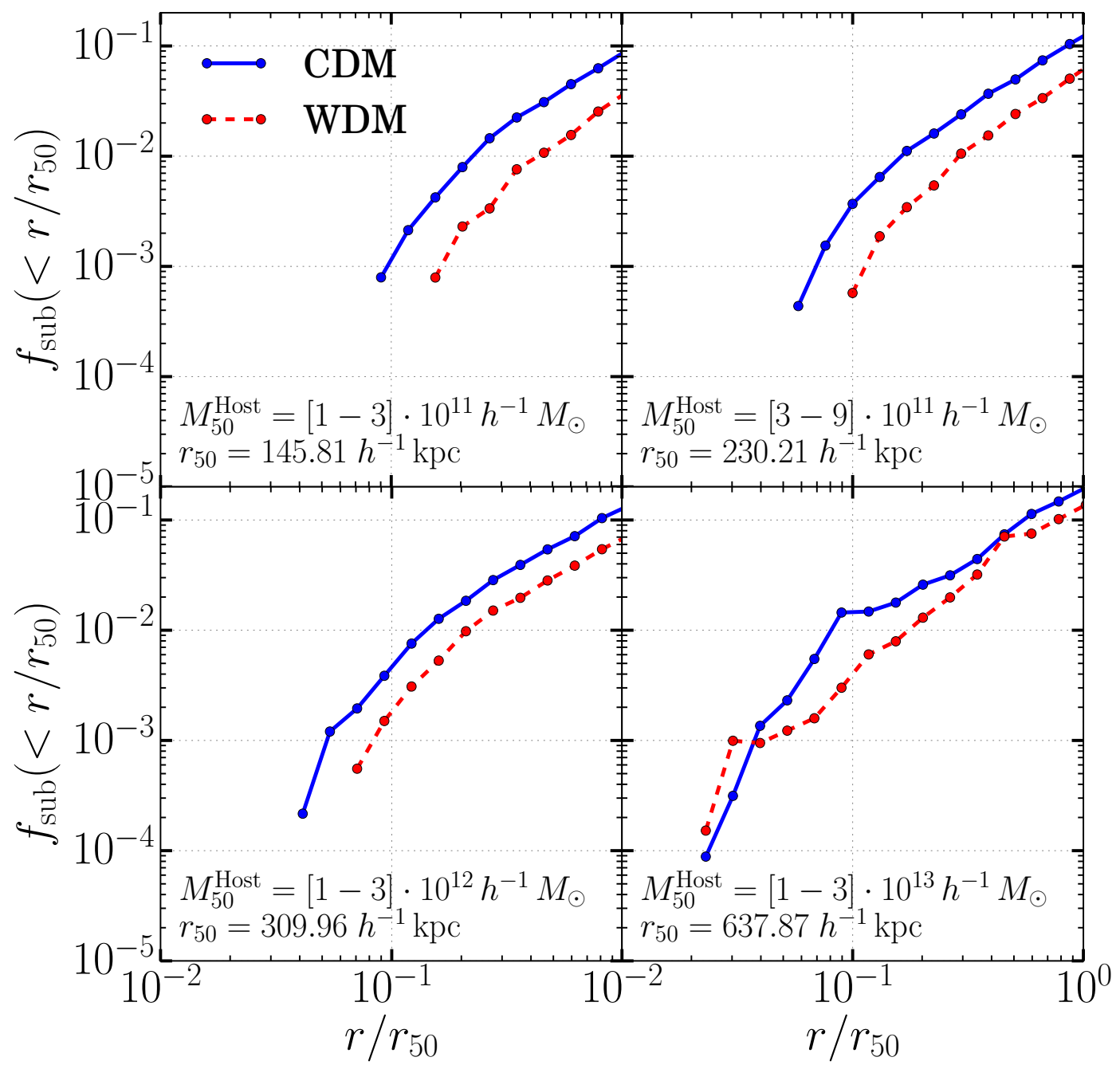

Figure 7. The mass fraction in substructures as a function of dimensionless radial distance from the halo centre, $r / r_{50}$, for COCO-COLD (solid blue) and COCO-WARM (dashed red) at $z=0$. The four different panels show results for stacks of host haloes of different mass as indicated in the legend. Only subhaloes with more than 300 particles are included. The value of $r_{50}$ quoted in each panel is the mean over all haloes in each (COCO-COLD) mass bin (the values are similar for COCO-WARM).

tions are averaged over 6 parent haloes with mass in the range $1 \times 10^{13} h^{-1} M_{\odot}<M_{50}^{\text {Host }}<3 \times 10^{13} h^{-1} M_{\odot}$, which are the best resolved in the simulation. The radial positions of the subhaloes are given in units of $r_{50}$. Only subhaloes resolved with more than 300 particles are included.

The dashed black lines in Fig. 6 give a fit to the CDM subhalo number density profiles using the Einasto profile (Einasto 1965; Navarro et al. 2004):

$$
\ln \left(\frac{n}{n_{-2}}\right)=-\frac{2}{\alpha}\left[\left(\frac{r}{r_{-2}}\right)^{\alpha}-1\right],
$$

where $n_{-2}$ is the characteristic number density at the scale radius $r=r_{-2}$. The values of $r_{-2}$ and shape parameter, $\alpha$, given in the legend. The fit is to COCO-COLD profile and the same curve is reproduced in the COCO-WARM panel.

The fit to the CDM subhalo profile also provides an excellent fit to the WDM profile, particularly at large radii. There are, however, differences of detail. The distribution of the more massive $\left(M_{\text {sub }}>10^{9} h^{-1} M_{\odot}\right)$ subhaloes beyond $r>0.2 r_{50}$ is very simi- lar in COCO-COLD and COCO-WARM. This regime is unaffected by the free streaming cutoff in COCO-WARM. Differences in the radial distribution of these more massive subhaloes can be attributed to small statistics: only six $\sim 10^{13} h^{-1} M_{\odot}$ haloes contribute to the average shown in Fig. 6. The profiles of the less massive subhaloes $\left(M_{\text {sub }}<10^{9} h^{-1} M_{\odot}\right)$ in WDM are somewhat steeper towards the centre than those in CDM. These subhaloes have masses below the cutoff scale, $M_{\mathrm{hm}}$, and their properties are affected by the cutoff. In particular, they form later than their CDM counterparts of the same mass today and, as a result, they have lower concentrations. These subhaloes experience more mass loss from tidal stripping after infall.

The approximate agreement of the subhalo radial distributions in COCO-COLD and COCO-WARM as well as the differences of detail are consistent with the analytic model proposed by Han et al. (2016). In this model, the $z=0$ radial number density of subhaloes, $n$, with mass, $m$, at distance, $R$, from the host halo centre 


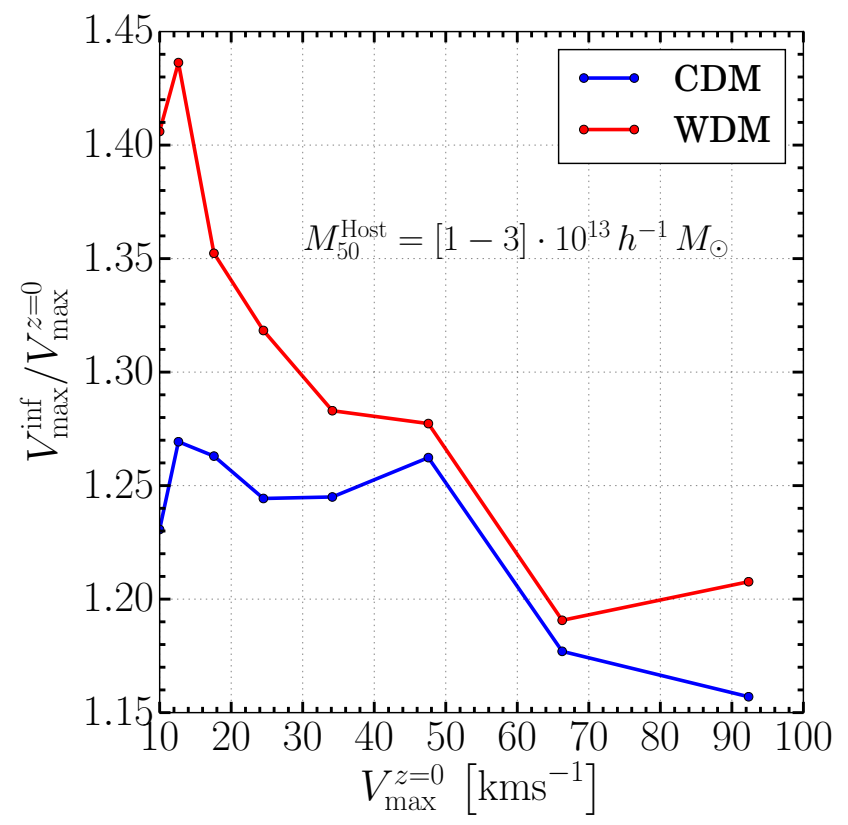

Figure 8. Ratio of the infall $\left(V_{\max }^{\text {inf }}\right)$ to present day $\left(V_{\max }^{z=0}\right)$ circular velocity, as a function of the present-day circular velocity. The results shown are for 6 stacked host haloes in the mass range $M_{50}^{\text {Host }}=[1-3] \cdot 10^{13} h^{-1} M_{\odot}$, using all subhaloes with more than 300 particles, located within $r_{50}$ of the host centre at $z=0$. The results for COCO-COLD are shown in blue and for COCO-WARM in red.

is given by:

$$
\frac{\mathrm{d} n(m, R)}{\mathrm{d} \ln m} \propto m^{-\alpha} R^{\gamma} \rho(R),
$$

where $\alpha$ is the slope of the subhalo mass function evaluated at $m$, $\rho(R)$ is the density profile of the host dark matter halo, $\gamma=\alpha \beta$, and $\beta \sim 1$ for an NFW density profile. The subhalo number density profile is suppressed relative to the host density profile by the factor $R^{\gamma}$. In COCO-COLD, the subhalo mass function follows a single power law but, in COCO-WARM, it has the same slope as in COCO-COLD for $M_{\text {sub }} \geqslant 10^{10} h^{-1} M_{\odot}$ and a shallower slope below that (see Fig. 3). A shallower slope results in a smaller value of $\alpha$ and therefore $\gamma$. Eq. 9 then predicts that, compared to CDM, the radial number density profile of small mass subhaloes should be suppressed less relative to the halo density profile for subhaloes. This explains why the two lowest subhalo mass bins in Fig. 6 exhibit steeper radial density profiles than the two highest mass bins.

An alternative way to examine the spatial distribution of substructures is to plot the fraction of mass within a given radius that is contained in substructures. This is shown in Fig. 7 for different ranges of host halo mass in COCO-COLD and COCO-WARM. The radial distributions have roughly the same shape in the two cases but the subhalo mass fractions are systematically lower in COCO-WARM than in COCO-COLD. In both cases, the substructure mass fractions are higher in more massive host haloes, particularly in the inner regions. For example, for host haloes of mass $M_{50}^{\text {Host }}=(1-3) \times 10^{13} h^{-1} M_{\odot}$ resolved substructures in COCOWARM contain about $10 \%$ of the halo mass within $r=r_{50}$, but only about $4 \%$ for host haloes of mass $M_{50}^{\text {Host }}=(1-3) \times 10^{11} h^{-1} M_{\odot}$. For reference, haloes (and subhaloes) contain $48 \%$ of the total mass in the simulation in COCO-WARM and $56 \%$ in COCO-COLD. In
CDM simulations these fractions depend on resolution, but not so in COCO-WARM where the cutoff in the power spectrum is resolved.

\subsection{Internal structure}

The density profiles of WDM haloes and subhaloes are cuspy and well described by the NFW (Navarro et al. 1997) form (Lovell et al. 2012; Schneider et al. 2012). However, the later formation times of WDM haloes of mass near the cutoff scale, compared to their CDM counterparts of the same mass, causes them to be less concentrated. In Bose et al. (2016a) we characterised the density and mass profiles of haloes in COCO-WARM over a range of halo masses and obtained the concentration-mass relation for WDM haloes (see also Ludlow et al. 2016). In summary, the density profiles of the largest haloes in COCO-WARM (roughly two orders of magnitude above $M_{\mathrm{hm}}$ ) are indistinguishable from their matched haloes in COCO-COLD, but the profiles of haloes of mass $M_{200}<7 \times 10^{10} h^{-1} M_{\odot}$ have systematically lower concentrations. In contrast with the power-law concentration-mass relation in $\mathrm{CDM}$, the relation in WDM turns over at below $\sim 10^{10} h^{-1} M_{\odot}$.

Calculating the concentration of subhaloes from their density profiles is not straightforward because the mass of a subhalo and therefore its "edge" are ambiguous. As Springel et al. (2008) showed, the size calculated by the SUBFIND algorithm (that is the radius of the saddle point in the density profile) coincides with the 'tidal' radius. Defining the concentration of the subhalo using this radius is not particularly useful because its value varies along the orbit. A more useful measure of subhalo concentration is the ratio $V_{\max } / r_{\max }$. In both WDM and CDM, the relation between $V_{\max }$ and $r_{\max }$ has a lower normalisation for subhaloes than for "field haloes" because of tidal stripping.

The fractional change in $V_{\max }$ between the moment of infall and the present day is shown in Fig. 8 for subhaloes (within $r_{50}$ ) of the most massive haloes in COCO-COLD and COCO-WARM, as a function of the present day maximum circular velocity, $V_{\max }^{z=0}$ (see also Diemand et al. 2007; Peñarrubia et al. 2008). The largest subhaloes, with $V_{\max }^{z=0} \geqslant 50 \mathrm{kms}^{-1}$, experience a reduction in $V_{\max }$ by a factor of $1.25-1.30$ after infall in both COCO-COLD and COCOWARM. Subhaloes of lower mass show significant differences between the two simulations. For example, at $V_{\max }^{z=0}=20 \mathrm{kms}^{-1}$, COCO-WARM subhaloes have experienced a reduction in $V_{\max }$ by a factor of $\sim 1.35$ since infall, compared to $\sim 1.25$ for COCO-COLD subhaloes.

The $r_{\max }-V_{\max }$ relations in COCO-COLD and COCO-WARM are shown in Fig. 9. For large subhaloes the two are very similar but the relations begin to diverge at values of $V_{\max }$ below of a few tens of kilometres per second, depending on the mass of the host halo. In this regime, haloes of a given $V_{\max }$ have larger $r_{\max }$ in COCO-WARM than in COCO-COLD and are therefore less concentrated. In both COCO-COLD and COCO-WARM subhaloes are more concentrated than field haloes, as a result of tidal stripping, but the difference between field haloes and subhaloes is larger in COCOWARM than in COCO-COLD. This reflects the greater tidal stripping experienced by COCO-WARM subhaloes, which have lower concentrations when they fall into the host halo. As a result, the concentrations of subhaloes in COCO-WARM increase more than those in COCO-COLD after infall. Overall, however, COCO-WARM subhaloes of a given mass (or $V_{\max }$ ) still have lower concentrations than COCO-COLD subhaloes. As noted in Hellwing et al. (2016), the importance of tidal stripping depends weakly on host halo mass: at a given $V_{\max }$, the reduction in $r_{\max }$ between field haloes and subhaloes is slightly larger for larger host halo masses 


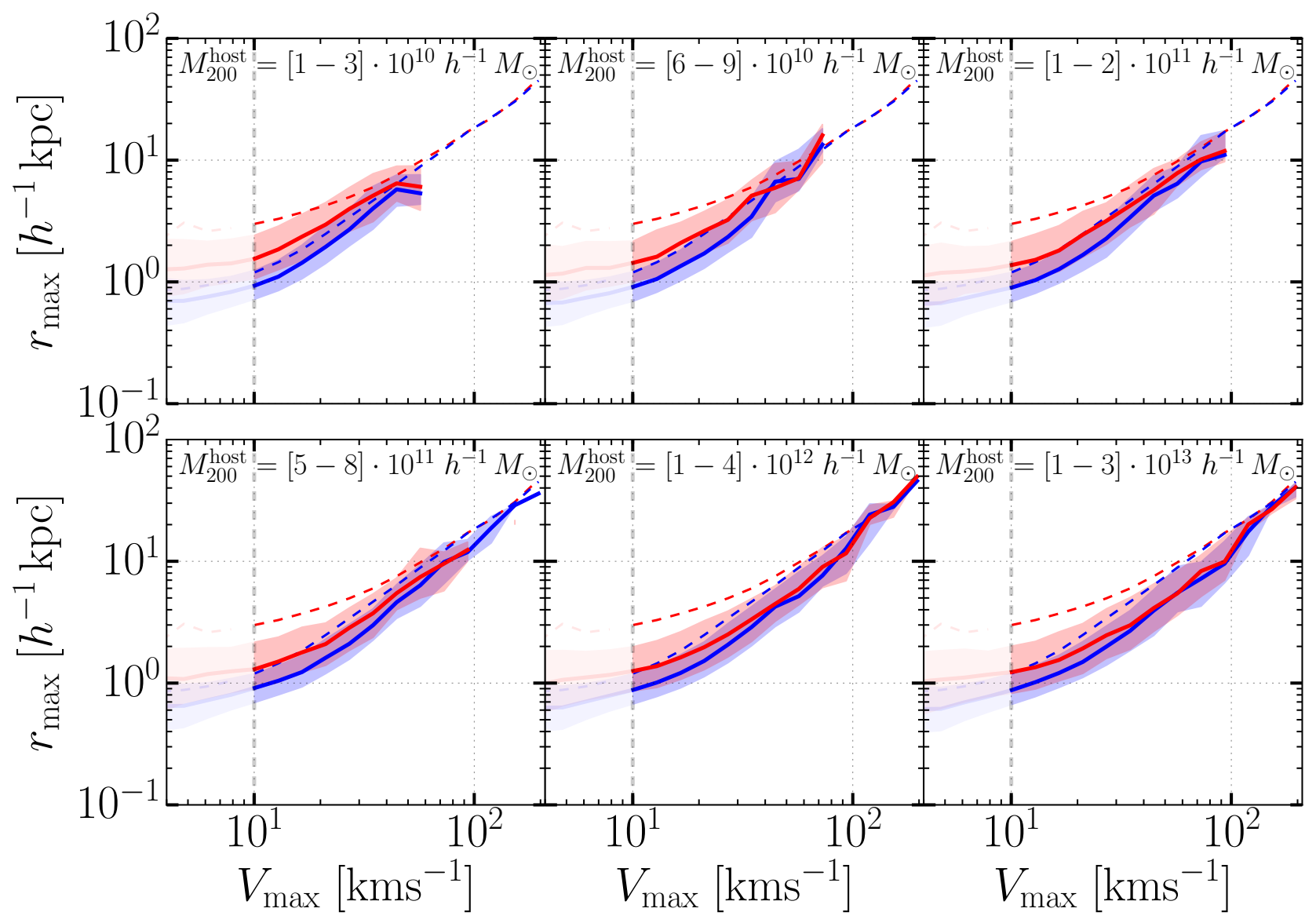

Figure 9. The subhalo $r_{\max }-V_{\max }$ relation in bins of parent halo mass (different panels) for COCO-COLD (blue) and COCO-WARM (red). Each panel shows results from stacking all host haloes within the given mass bin. The solid line in each case shows the median relation in the stack, whereas the shaded regions correspond to the $16^{\text {th }}$ and $84^{\text {th }}$ percentiles. The dashed lines show the median relation for "field" haloes in each case. The plots are made translucent for $V_{\max }<10 \mathrm{kms}^{-1}$, below which resolution effects become increasingly important (see Appendix A in Hellwing et al. 2016).

\section{GALAXY FORMATION WITH WARM DARK MATTER}

Our analysis so far has been restricted to the dark matter properties of a $3.3 \mathrm{keV}$ thermal relic or, equivalently, a $7 \mathrm{keV}$ sterile neutrino with leptogenesis parameter, $L_{6}=8.66$, the "coldest" $7 \mathrm{keV}$ sterile neutrino compatible with the observed $3.5 \mathrm{keV} \mathrm{X}$-ray line. While future gravitational lensing surveys may provide a direct way to measure the mass function of dark matter substructures and thus distinguish CDM from WDM (Vegetti \& Koopmans 2009; Li et al. 2016), it is worth investigating whether CDM and WDM can be distinguished with current observations. At high redshift, the observed clumpiness of the Lyman- $\alpha$ forest has been used to rule out WDM models with thermally produced particles of mass $m_{\text {WDM }} \leqslant 3.3 \mathrm{kev}$ (Viel et al. 2013). As mentioned in Section 1, constraints obtained from the Lyman- $\alpha$ forest depend on assumptions for the thermal history of the IGM.

To compare the models with other astronomical data we need to populate the dark matter subhaloes with galaxies. This can be done in three ways. One is to use empirical prescriptions such as "abundance matching" (see e.g. Reed et al. 2015) but Sawala et al. (2015) have shown that this technique breaks down for halo masses $<10^{10} h^{-1} M_{\odot}-$ precisely the scale of interest in WDM. The failure of abundance matching in this regime is due to the physics of reionisation, which inhibits the formation of stars in low mass haloes after the epoch of reionisation, and to the effects of supernovae feedback. A second technique are hydrodynamical simulations but these are computationally expensive and, to date, only limited WDM cosmological simulations have been carried out (e.g. Herpich et al. 2014; Carucci et al. 2015; González-Samaniego et al. 2016). The third approach, the one we use here, is semianalytical modelling of galaxy formation, a flexible and powerful technique that requires only modest computational resources. We apply the Durham semi-analytic model, GALFORM, to halo merger trees in COCO-COLD and COCO-WARM. This model includes detailed treatments of gas cooling, star formation, metal production, galaxy mergers and instabilities, black hole growth and feedback from energy released by stellar evolution and AGN. This model was previously used by Kennedy et al. (2014) to set a lower limit to the mass of thermally produced WDM particles.

Details of the modelling in GALFORM may be found in the papers presenting the original formulation of the model (Cole et al. 2000) and its latest version (Lacey et al. 2016). Here we use this latest model for both COCO-COLD and COCO-WARM without any modification $^{2}$

\footnotetext{
2 Kennedy et al. (2014) found that a small modification to one of the supernovae feedback parameters was required for their WDM models to produce acceptable $b_{J}$ and $K$-band luminosity functions at $z=0$. The particle mass
} 

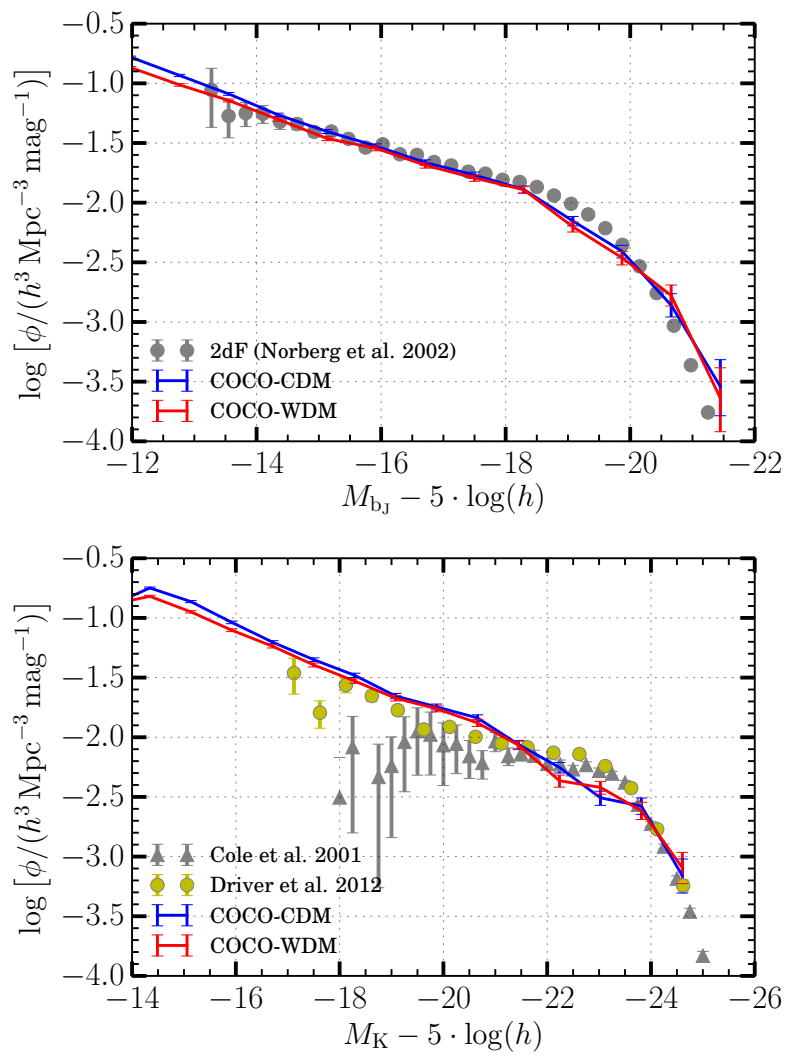

Figure 10. The $z=0 b_{J}$ - (upper panel) and $K$-band (lower panel) luminosity functions from GALFORM applied to halo merger trees constructed from the COCO-COLD (blue) and COCO-WARM (red) simulations (see text for details). The symbols represent observational data from Norberg et al. 2002, Cole et al. 2001 and Driver et al. 2012.

\subsection{Field and satellite luminosity functions}

The galaxy luminosity functions in the $b_{J}$ and $K$-bands in COCOCOLD (see also Guo et al. 2015) and COCO-WARM are compared with observational data in Fig. 10. The parameters controlling supernova feedback in GALFORM are calibrated to reproduce the observed luminosity functions at $z=0$ in these bands. The two models predict essentially identical luminosity functions except at faint magnitudes where there are slightly fewer galaxies in WDM, as a result of the lower abundance of small mass haloes in this model. At the faintest magnitudes plotted the difference is only about $25 \%$, smaller than the observational error bars. Due to the small volume of the COCO high resolution region, there are only a few bright galaxies in the simulations, as reflected in the large Poisson errors bars at the brightest magnitudes.

Fainter galaxies than those plotted in Fig. 10 are only detectable in the nearby Universe, particularly in the Local Group. Only a few tens of satellites have been discovered orbiting the haloes of the Milky Way and Andromeda. This number is much smaller than the number of small subhaloes seen in CDM simulations of galactic haloes and this observation has often been used to motivate WDM models. In fact, it has been shown, using a variety

in the model we are considering here, $3.3 \mathrm{keV}$, is sufficiently large that not even this minor modification is required. of modelling techniques, that most of these small subhaloes are not able to make a visible galaxy either because their gas is heated by reionisation or expelled altogether by supernovae explosions. The earliest explicit demonstration of this simple physics was provided by the semi-analytic models of Bullock et al. (2000) and Benson et al. (2002) and the latest by the APOSTLE hydrodynamic simulations of Sawala et al. (2016).

In fact, WDM models can be constrained by the observed number of faint satellites because if the particle mass is too small not enough subhaloes would form to account even for the observed number of satellites in the Milky Way (which may be underestimated because of incompleteness in current surveys). Kennedy et al. (2014) used this argument to set constraints on the allowed masses of thermally produced WDM particles. These constraints depend on the assumed mass of the Milky Way halo because the number of subhaloes scales with the mass of the parent halo (as seen, for example, in Fig. 4 above). Kennedy et al. (2014) find that all thermal WDM particle masses are ruled out (at 95\% confidence) if the halo of the Milky Way has a mass smaller than $7.7 \times 10^{11} h^{-1} M_{\odot}$, while if the mass of the Galactic halo is greater than $1.3 \times 10^{12} h^{-1} M_{\odot}$ only WDM particle masses larger than $2 \mathrm{keV}$ are allowed.

We perform a similar analysis here. Fig. 11 shows the cumulative number of satellites as a function of $V$-band magnitude, $\mathrm{M}_{V}$, in COCO-COLD and COCO-WARM for three bins of host halo mass, with median values of $1.2 \times 10^{12}, 1.6 \times 10^{12}$ and $2.0 \times 10^{12} h^{-1} M_{\odot}$. The luminosity function of satellites in the Milky Way, shown by the black solid lines in the figure, include the 11 classical satellites. For $M_{V}<-11$, the data has been obtained from the direct observations of McConnachie (2012). The abundance of ultra-faint satellites found in the SDSS has been corrected for incompleteness and partial sky coverage by Koposov et al. (2008). The faint objects recently discovered by DES (Bechtol et al. 2015; Drlica-Wagner et al. 2015) are represented by the black diamond following the analysis of (Jethwa et al. 2016) who find that of the 14 newly-detected satellites, 12 have $>50 \%$ probability of having been brought in as satellites of the LMC (at 95\% confidence). Jethwa et al. (2016) extrapolate the detected population to estimate that the Milky Way should have $\sim 180$ satellites within $300 \mathrm{kpc}$, in addition to $70_{-40}^{+30}$ Magellanic satellites in the $V$-band magnitude range $-7<M_{V}<-1$ (68\% confidence). All observational error bars in Fig. 11 are Poisson errors, with volume corrections made where appropriate. In order to match the observational selection, only satellites within $300 \mathrm{kpc}$ of the central galaxy are included.

The satellite luminosity functions are very similar in COCO-COLD and COCO-WARM. Only at magnitudes fainter than $\mathrm{M}_{\mathrm{V}} \simeq \quad-4$ does the number of satellites in COCO-WARM begin to drop below the number in COCOCOLD. The models agree with the data so long as the Milky Way halo mass is $M_{200}^{\text {host }} \lesssim 1.2 \times 10^{12} h^{-1} M_{\odot}$. For $M_{200}^{\text {host }} \sim 1.6 \times 10^{12} h^{-1} M_{\odot}$, both COCO-COLD and COCOWARM significantly overpredict the number of satellites even at relatively bright magnitudes, $M_{V} \sim-10$, where the known sample is unlikely to be significantly incomplete. There is a significant difference in the abundance of satellites with magnitude $M_{V} \sim-1$, the regime where DES has just begun to uncover ultra-faint dwarf galaxies. These new data could potentially be used to set strong constraints on the mass of the WDM particle. It must be borne in mind that the exact location of this (extrapolated) DES data point depends on the DES selection function, detection efficiency, and assumptions made about isotropy in the distribution of Milky Way 


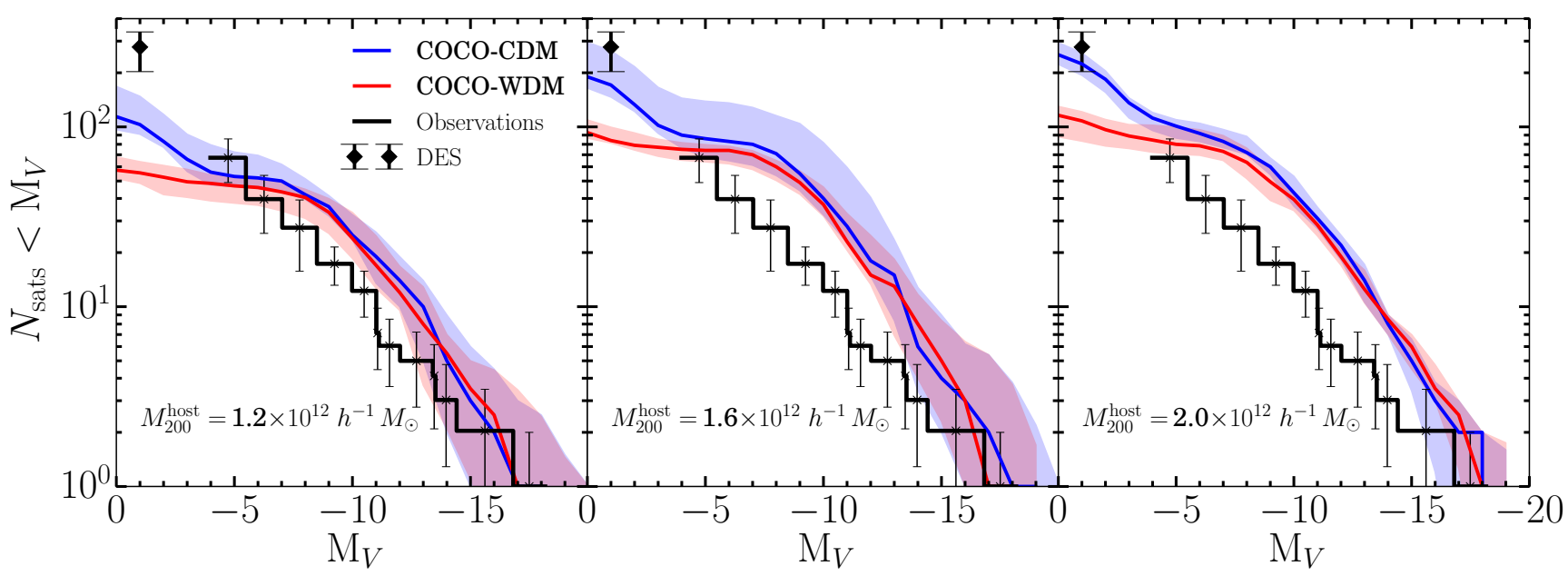

Figure 11. The cumulative $V$-band luminosity function of satellites within $280 \mathrm{kpc}$ of the centre of Milky Way-like haloes in COCO-COLD (blue) and COCOWARM (red). Each panel shows the average luminosity function for host haloes in three bins of mass, $M_{200}=1-3 \times 10^{12} h^{-1} M_{\odot}, 1.5-1.7 \times$ $10^{12} h^{-1} M_{\odot}$ and $1.8-2.1 \times 10^{12} h^{-1} M_{\odot}$. The values quoted in the legend are the medians in each bin. The shaded regions indicate the 5th and 95th percentiles. The black step function shows the data for the Milky Way. For $\mathrm{M}_{\mathrm{V}} \geqslant-11$, the data has been corrected for incompleteness and sky coverage by Koposov et al. 2008. For $\mathrm{M}_{\mathrm{V}}<-11$, the histogram shows the direct observational data from McConnachie 2012. The black diamond is an extrapolation of the luminosity function to $M_{\mathrm{V}} \sim-1$ after including the ultra-faint dwarf satellites recently discovered by DES (Jethwa et al. 2016).

satellites. Furthermore, although we have used a well-tested, stateof-the-art model of galaxy formation, these conclusions depend on assumptions in the model, particularly on the treatment of reionisation and supernovae feedback (Hou et al. 2016).

\subsection{Evolution of the UV luminosity function}

The evolution of luminosity function in the rest-frame UV traces the star formation history in the Universe. Although still rather scarce and uncertain, data now exist out to redshift $z \sim 10$. Since the formation of structure begins later in WDM models than in $\mathrm{CDM}$ we might naïvely expect to find fewer star-forming galaxies at high redshift in COCO-WARM than in COCO-COLD. The actual predictions are shown in Fig. 12, which reveals that, in fact, the result is exactly the opposite: at $z>5$, the UV luminosity function has a higher amplitude in COCO-WARM than in COCO-COLD. The reason for this is that, in CDM, supernovae-driven winds limit the reservoir of cold, potentially star-forming, gas in low-mass galaxies at early times. The brightest UV galaxies at high redshift tend to be starbursts triggered by mergers of these relatively gas poor galaxies (Lacey et al. 2016). By contrast in WDM, the first galaxies that collapse are more massive than their CDM counterparts and more gas rich, thus producing brighter starbursts when they merge. This makes the formation of bright galaxies at high redshift more efficient in WDM than in CDM.

Although both COCO-COLD and COCO-WARM somewhat underpredict current observations at $z>7$, the data have large statistical, and potentially systematic errors since these objects are rare and current surveys cover relatively small volumes. If anything, COCO-WARM is closer to the data than COCO-COLD. This result is broadly consistent with those of Dayal et al. (2015) who used a simpler model of galaxy formation to derive the UV luminosity function in WDM models. The existence of a population of star-forming galaxies in COCO-WARM at $z>8$ has the additional benefit that enough ionising photons are produced at early times to reionise the universe by $z \simeq 8$, as required by the optical depth to reionisation inferred from Planck (Planck Collaboration et al. 2014). Reionisation in WDM models is discussed in detail by Bose et al. (2016b).

Fig. 13 helps visualise the counter-intuitive result just described. In the left panel we plot, as a function of redshift, the stellar mass growth, $M_{\star}(z)$, averaged over all galaxies with $1 \times 10^{7} h^{-1} M_{\odot}<M_{\star}<5 \times 10^{7} h^{-1} M_{\odot}$ at $z=7$ in COCO-WARM (red) and COCO-COLD (blue). This range of stellar mass corresponds to galaxies brighter than $M_{\mathrm{AB}}(\mathrm{UV}) \leqslant-17$ in Fig. 12. $M_{\star}(z)$ is normalised to the stellar mass of the galaxy at $z=7, M_{\star}(z=7)$. The stellar mass assembly in COCO-WARM is delayed relative to that in COCO-COLD because the earliest progenitors form later in COCO-WARM. For $12>z>8$, the build-up of stellar mass is gradual in both COCO-COLD and COCO-WARM, although the slope of the mass growth is steeper in the latter i.e., more stellar mass builds up per unit redshift in COCO-WARM than in COCO-COLD. This is supported by the right panel of Fig. 13, which shows the evolution of the specific star formation rate ( $\mathrm{SSFR}$ ) of these galaxies. COCO-WARM galaxies exhibit systematically higher sSFRs than COCO-COLD up to $z=8$. This is in consistent with our earlier suggestion that COCO-WARM galaxies are formed out of more gas-rich progenitors. Mergers of these gas-rich progenitors allows galaxies in COCO-WARM to "catch-up" with those in COCOCOLD after their delayed start of star formation.

At $z \leqslant 3$ the UV luminosity functions in COCO-COLD and COCO-WARM are indistinguishable even down to magnitudes as faint as $M_{\mathrm{AB}}(\mathrm{UV}) \approx-10$. These galaxies form in haloes of mass $\sim 10^{10} h^{-1} M_{\odot}$, the scale at which the subhalo mass functions in COCO-WARM just begin to diverge from those in COCO-COLD (see Fig. 3). At even fainter magnitudes $\left(M_{\mathrm{AB}}(\mathrm{UV}) \geqslant-7\right.$, not shown), the luminosity function for COCO-WARM is strongly suppressed relative to COCO-COLD but these magnitudes are far below the detection limits of even the JWST.

We have checked that the results in this section are not sensitive to the specific version of the GALFORM model used. The result 


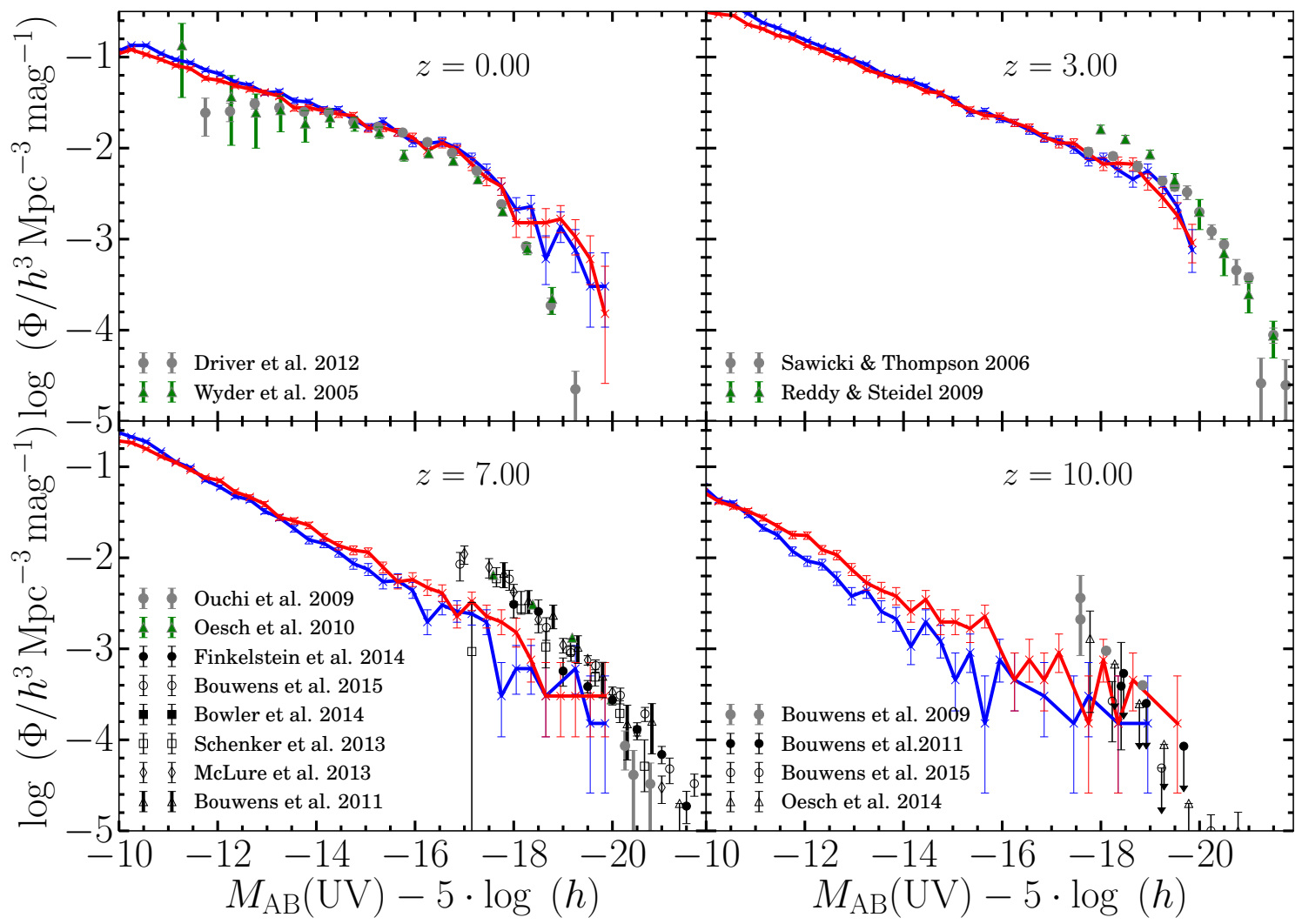

Figure 12. The evolution of the UV luminosity function of all galaxies (centrals and satellites) for $z=0,3,7,10$. The red lines represents COCO-WARM and the blue COCO-COLD, with Poisson errors plotted. The colour symbols with errorbars show observational data taken from Driver et al. 2012, Wyder et al. 2005, Sawicki \& Thompson 2006, Reddy \& Steidel 2009, Ouchi et al. 2009, Oesch et al. 2010, Bouwens et al. 2009, Bouwens et al. $2011 \mathrm{a}$,b, Schenker et al. 2013, McLure et al. 2013, Finkelstein et al. 2015, Bowler et al. 2014, Oesch et al. 2014 and Bouwens et al. 2015.

in Fig. 12 holds for the Gonzalez-Perez et al. (2014) model, with and without the assumption of gradual ram-pressure stripping of hot gas in satellite galaxies (Font et al. 2008), as well as for the Hou et al. (2016) model in which supernova feedback is much weaker than in our standard model at high- $z$ and becomes progressively stronger at lower redshift. The simpler model by Dayal et al. (2015) is forced to match the observed UV luminosity function at high- $z$ and cannot, by construction, exhibit any differences between WDM and CDM.

\subsection{Other galactic observables}

In addition to the galaxy properties just discussed, we have explored a number of others, such as colour and metallicity distributions; sizes; the Tully-Fisher relation; and spatial clustering. We do not find any significant, potentially observable differences between COCO-COLD and COCO-WARM. This conclusion reinforces the point that, apart from the details discussed in Section 4.1 and 4.2, galaxy formation is very similar in CDM and in a $7 \mathrm{keV}$ sterile neutrino (or a $3.3 \mathrm{keV}$ thermal WDM) model.

\section{SUMMARY AND CONCLUSIONS}

Using the Copernicus Complexio (COCO) high resolution dark matter simulations (Hellwing et al. 2016), we have carried out a thor- ough investigation of the small-scale differences between CDM and a model with the same phases but with a cutoff in the initial power spectrum of fluctuations that can be interpreted either as that of the "coldest" sterile neutrino model compatible with the recently detected $3.5 \mathrm{keV} \mathrm{X}$-ray line or as a $3.3 \mathrm{keV}$ thermal particle model.

The subhalo mass functions in the two models (COCO-COLD and COCO-WARM) are identical at high masses but the number density of COCO-WARM subhaloes begins to fall below that of COCOCOLD subhaloes at $\sim 5 \times 10^{9} h^{-1} M_{\odot}$ and is very strongly suppressed below $\sim 2.5 \times 10^{8} h^{-1} M_{\odot}$, the half-mode mass in the initial power spectrum, When the number counts are expressed in units of parent halo properties such as $M_{\text {sub }} / M_{200}$ and $V_{\max } / V_{200}$, we find that the subhalo mass and $V_{\max }$ functions in COCO-COLD follow a nearly universal profile with little dependence on host halo mass, confirming earlier results (Moore et al. 1999; Kravtsov et al. 2004; Zheng et al. 2005; Springel et al. 2008; Weinberg et al. 2008; Wang et al. 2012; Cautun et al. 2014). This self-similar behaviour does not occur in COCO-WARM.

The normalised radial distribution of subhaloes in both models is independent of the mass of the subhaloes. In the case of COCO-WARM this behaviour extends to the smallest subhaloes in the simulation, with $M_{\text {sub }} \simeq 10^{8} h^{-1} M_{\odot}$, although there is a slight steepening of their profile in the very central parts of the halo. Our findings extend the results from the AQUARIUS and PHOENIX simulations (Springel et al. 2008; Gao et al. 2012) and lend support to the model proposed by Han et al. (2016) in which the mass in- 

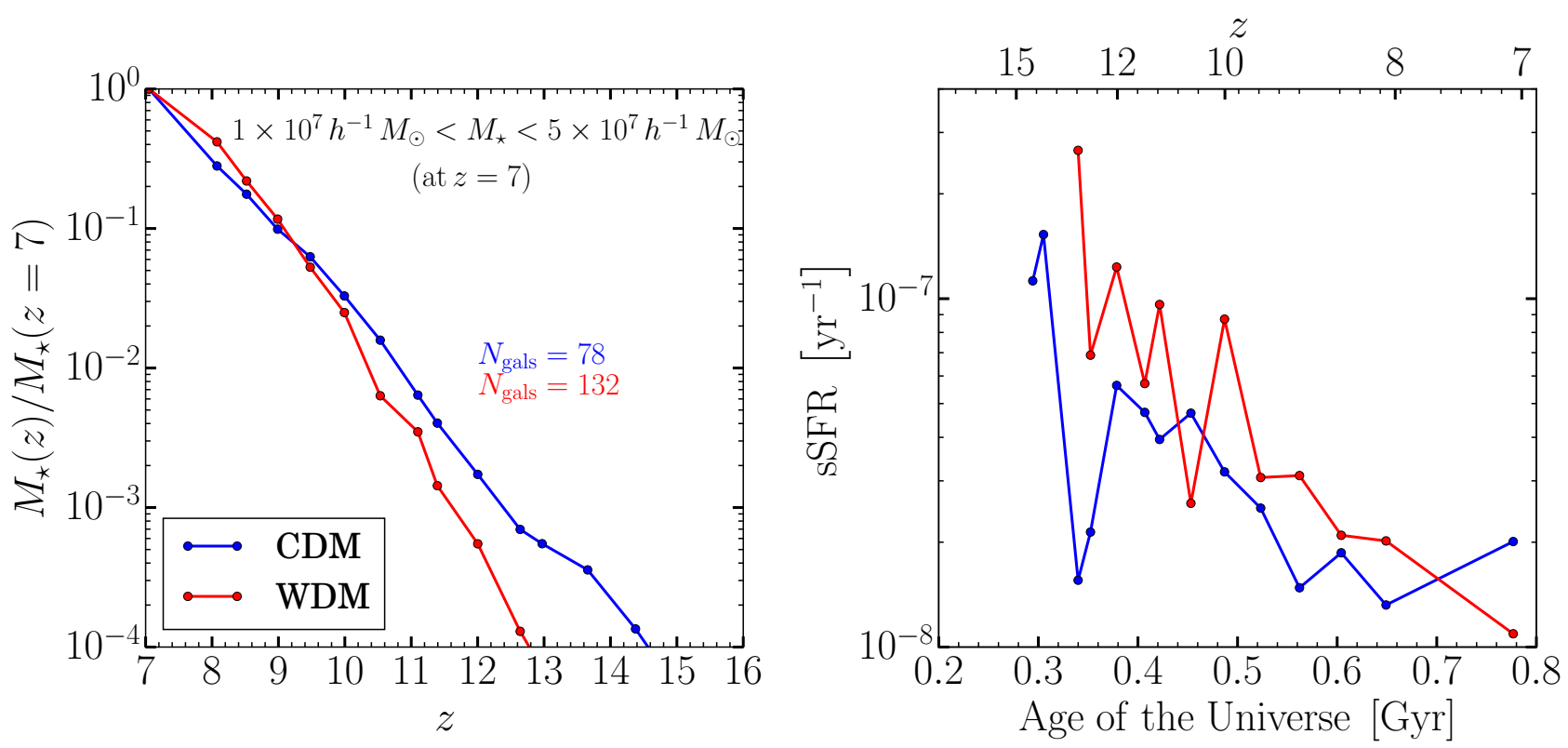

Figure 13. Left panel: the average stellar mass growth of all galaxies with mass $1 \times 10^{7} h^{-1} M_{\odot}<M_{\star}<5 \times 10^{7} h^{-1} M_{\odot}$ in COCO-COLD (blue) and COCO-WARM (red). The mass as a function of redshift, $M_{\star}(z)$, is normalised to the final stellar mass at $z=7$. The number of galaxies averaged over in each simulation is indicated in the plot with the corresponding colour. Right panel: the specific star formation history as a function of the age of the Universe. The galaxies averaged over are the same as in the left panel.

variance of the radial distribution results from the effects of tidal stripping. The radial density profiles are well approximated by either the NFW or Einasto forms.

Subhaloes in both COCO-COLD and COCO-WARM are cuspy and follow the NFW form. Small-mass WDM haloes, in general, are less concentrated than CDM haloes of the same mass reflecting their later formation epoch. For WDM subhaloes with $V_{\max }^{z=0} \leqslant 50 \mathrm{kms}^{-1}$, the difference is exacerbated because their lower concentrations make them more prone to tidal stripping after they are accreted into the host halo.

In order to check if the two models can be distinguished with current observations, we populated the haloes with model galaxies whose properties were calculated using the Durham semi-analytic galaxy formation model, GALFORM. We used the latest version of GALFORM (Lacey et al. 2016) without needing to adjust any model parameters for COCO-WARM. The COCO-COLD and COCOWARM $b_{J}$ and $K$-band luminosity functions at $z=0$ are very similar, except at the faintest end where there are slightly fewer dwarfs in COCO-WARM; both models give a good match to the observations. The same is true at the fainter magnitudes represented by the satellites of the Milky Way: both models agree with current data provided the mass of the Milky Way halo is less than $M_{200}=1.2 \times 10^{12} h^{-1} M_{\odot}$. The two models could be distinguished if the satellite luminosity function faintwards of $M_{V} \sim-3$ or -4 could be measured reliably because COCO-WARM predicts about half the number of satellites as COCO-COLD at these luminosities.

The only other significant difference that we have found between COCO-COLD and COCO-WARM is in the UV luminosity function at $z>7$ where there are more UV-bright galaxies in COCO-WARM than in COCO-COLD. The qualitative difference between the UV luminosity functions in COCO-WARM and COCO-
COLD is not strongly affected by the treatment of baryon physics in the GALFORM semi-analytic model. This difference, however (a factor of $\sim 2$ at $z>8$ ), cannot be detected with current data. None of the other galaxy properties we examined: colour and metallicity distributions, scaling relations, spatial clustering, etc. differ in the two models in the regime where these properties can be studied observationally.

In summary, the "coldest" sterile neutrino model compatible with the identification of the recently detected $3.5 \mathrm{keV} \mathrm{X}$-ray line as resulting from the decay of these particles cannot, at present, be distinguished from a CDM model by observations of galaxies, ranging from the satellites of the Milky Way to the brightest starbursts at $z=10$. The two models are drastically different in their dark matter properties on subgalactic scales where the sterile neutrino model predicts orders of magnitude fewer subhaloes of mass $M \lesssim 10^{8} h^{-1} M_{\odot}$ than produced in CDM. These small masses are, in principle, accessible to gravitational lensing (Vegetti \& Koopmans 2009; Li et al. 2016), and it is to be hoped that future surveys will be able conclusively to rule out one or the other or both of these models.

\section{ACKNOWLEDGEMENTS}

We are grateful to Cedric Lacey for comments and help with GALFORM. This work was supported by: the European Research Council grants GA 267291 (Cosmiway) and 646702 (CosTesGrav), the Science and Technology Facilities Council [grant number ST/F001166/1, ST/I00162X/1, ST/K501979/1] and the Polish National Science Center under contract \#UMO2012/07/D/ST9/02785. This work used the DiRAC Data Centric system at Durham University, operated by the Institute for 
Computational Cosmology on behalf of the STFC DiRAC HPC Facility (www.dirac.ac.uk). This equipment was funded by BIS National E-infrastructure capital grant ST/K00042X/1, STFC capital grant ST/H008519/1, and STFC DiRAC Operations grant ST/K003267/1 and Durham University. DiRAC is part of the National E-Infrastructure. Some simulations used here were computed at the Interdiciplinary Centre for Mathematical \& Computational Modelling at University of Warsaw with the support of the HPC Infrastructure for Grand Challenges of Science and Engineering Project, co-financed by the European Regional Development Fund under the Innovative Economy Operational Programme. This work is also part of the D-ITP consortium, a program of the Netherlands Organisation for Scientific Research (NWO) that is funded by the Dutch Ministry of Education, Culture and Science (OCW).

\section{REFERENCES}

Anderson M. E., Churazov E., Bregman J. N., 2015, MNRAS, 452, 3905

Angulo R. E., Hahn O., Abel T., 2013, MNRAS, 434, 3337

Asaka T., Shaposhnikov M., 2005, Physics Letters B, 620, 17

Baur J., Palanque-Delabrouille N., Yèche C., Magneville C., Viel M., 2016, JCAP, 8, 012

Bechtol K., et al., 2015, ApJ, 807, 50

Benson A. J., Frenk C. S., Lacey C. G., Baugh C. M., Cole S., 2002, MNRAS, 333, 177

Bode P., Ostriker J. P., Turok N., 2001, ApJ, 556, 93

Bose S., Hellwing W. A., Frenk C. S., Jenkins A., Lovell M. R., Helly J. C., Li B., 2016a, MNRAS, 455, 318

Bose S., Frenk C. S., Hou J., Lacey C. G., Lovell M. R., 2016b, MNRAS, 463,3848

Bouwens R. J., et al., 2009, ApJ, 690, 1764

Bouwens R. J., et al., 2011a, Nature, 469, 504

Bouwens R. J., et al., 2011b, ApJ, 737, 90

Bouwens R. J., et al., 2015, ApJ, 803, 34

Bowler R. A. A., et al., 2014, MNRAS, 440, 2810

Boyarsky A., Ruchayskiy O., Shaposhnikov M., 2009, Annual Review of Nuclear and Particle Science, 59, 191

Boyarsky A., Ruchayskiy O., Iakubovskyi D., Franse J., 2014, Physical Review Letters, 113, 251301

Bulbul E., Markevitch M., Foster A., Smith R. K., Loewenstein M., Randall S. W., 2014, ApJ, 789, 13

Bullock J. S., Kravtsov A. V., Weinberg D. H., 2000, ApJ, 539, 517

Carucci I. P., Villaescusa-Navarro F., Viel M., Lapi A., 2015, JCAP, 7, 047

Cautun M., Hellwing W. A., van de Weygaert R., Frenk C. S., Jones B. J. T., Sawala T., 2014, MNRAS, 445, 1820

Cole S., Lacey C. G., Baugh C. M., Frenk C. S., 2000, MNRAS, 319, 168

Cole S., et al., 2001, MNRAS, 326, 255

Cole S., et al., 2005, MNRAS, 362, 505

Colín P., Avila-Reese V., Valenzuela O., 2000, ApJ, 542, 622

Colín P., Avila-Reese V., González-Samaniego A., Velázquez H., 2015, ApJ, 803, 28

Colless M., et al., 2001, MNRAS, 328, 1039

Dayal P., Mesinger A., Pacucci F., 2015, ApJ, 806, 67

Diemand J., Kuhlen M., Madau P., 2007, ApJ, 667, 859

Driver S. P., et al., 2012, MNRAS, 427, 3244

Drlica-Wagner A., et al., 2015, ApJ, 813, 109

Einasto J., 1965, Trudy Astrofizicheskogo Instituta Alma-Ata, 5, 87

Eisenstein D. J., et al., 2005, ApJ, 633, 560

Finkelstein S. L., et al., 2015, ApJ, 810, 71

Font A. S., et al., 2008, MNRAS, 389, 1619

Frenk C. S., White S. D. M., 2012, Annalen der Physik, 524, 507

Gao L., Navarro J. F., Frenk C. S., Jenkins A., Springel V., White S. D. M., 2012, MNRAS, 425, 2169

Garzilli A., Boyarsky A., Ruchayskiy O., 2015, preprint, (arXiv:1510.07006)
Gonzalez-Perez V., Lacey C. G., Baugh C. M., Lagos C. D. P., Helly J., Campbell D. J. R., Mitchell P. D., 2014, MNRAS, 439, 264

González-Samaniego A., Avila-Reese V., Colín P., 2016, ApJ, 819, 101

Guo Q., Cooper A. P., Frenk C., Helly J., Hellwing W. A., 2015, MNRAS, 454,550

Han J., Cole S., Frenk C. S., Jing Y., 2016, MNRAS, 457, 1208

Hawkins E., et al., 2003, MNRAS, 346, 78

Hellwing W. A., Frenk C. S., Cautun M., Bose S., Helly J., Jenkins A., Sawala T., Cytowski M., 2016, MNRAS, 457, 3492

Herpich J., Stinson G. S., Macciò A. V., Brook C., Wadsley J., Couchman H. M. P., Quinn T., 2014, MNRAS, 437, 293

Hobbs A., Read J. I., Agertz O., Iannuzzi F., Power C., 2016, MNRAS, 458, 468

Hooper D., Goodenough L., 2011, Physics Letters B, 697, 412

Horiuchi S., Bozek B., Abazajian K. N., Boylan-Kolchin M., Bullock J. S., Garrison-Kimmel S., Onorbe J., 2016, MNRAS, 456, 4346

Hou J., Frenk C. S., Lacey C. G., Bose S., 2016, MNRAS,

Jethwa P., Erkal D., Belokurov V., 2016, MNRAS, 461, 2212

Kennedy R., Frenk C., Cole S., Benson A., 2014, MNRAS, 442, 2487

Klypin A. A., Trujillo-Gomez S., Primack J., 2011, ApJ, 740, 102

Knebe A., Arnold B., Power C., Gibson B. K., 2008, MNRAS, 386, 1029

Koposov S., et al., 2008, ApJ, 686, 279

Kravtsov A. V., Berlind A. A., Wechsler R. H., Klypin A. A., Gottlöber S., Allgood B., Primack J. R., 2004, ApJ, 609, 35

Lacey C. G., et al., 2016, MNRAS, 462, 3854

Li R., Frenk C. S., Cole S., Gao L., Bose S., Hellwing W. A., 2016, MNRAS, 460, 363

Lovell M. R., et al., 2012, MNRAS, 420, 2318

Lovell M. R., Frenk C. S., Eke V. R., Jenkins A., Gao L., Theuns T., 2014, MNRAS, 439, 300

Lovell M. R., et al., 2016, MNRAS, 461, 60

Ludlow A. D., Navarro J. F., Springel V., Jenkins A., Frenk C. S., Helmi A., 2009, ApJ, 692, 931

Ludlow A. D., Bose S., Angulo R. E., Wang L., Hellwing W. A., Navarro J. F., Cole S., Frenk C. S., 2016, MNRAS, 460, 1214

Macciò A. V., Ruchayskiy O., Boyarsky A., Muñoz-Cuartas J. C., 2013, MNRAS, 428, 882

Malyshev D., Neronov A., Eckert D., 2014, Phys. Rev. D, 90, 103506

McConnachie A. W., 2012, AJ, 144, 4

McLure R. J., et al., 2013, MNRAS, 432, 2696

Moore B., Ghigna S., Governato F., Lake G., Quinn T., Stadel J., Tozzi P., 1999, ApJ, 524, L19

Navarro J. F., Frenk C. S., White S. D. M., 1997, ApJ, 490, 493

Navarro J. F., et al., 2004, MNRAS, 349, 1039

Norberg P., et al., 2002, MNRAS, 336, 907

Oesch P. A., et al., 2010, ApJ, 709, L16

Oesch P. A., et al., 2014, ApJ, 786, 108

Ouchi M., et al., 2009, ApJ, 706, 1136

Peñarrubia J., Navarro J. F., McConnachie A. W., 2008, ApJ, 673, 226

Planck Collaboration et al., 2014, A\&A, 571, A1

Reddy N. A., Steidel C. C., 2009, ApJ, 692, 778

Reed D. S., Schneider A., Smith R. E., Potter D., Stadel J., Moore B., 2015, MNRAS, 451, 4413

Riemer-Sørensen S., 2016, A\&A, 590, A71

Sawala T., et al., 2015, MNRAS, 448, 2941

Sawala T., et al., 2016, MNRAS, 457, 1931

Sawicki M., Thompson D., 2006, ApJ, 642, 653

Schenker M. A., et al., 2013, ApJ, 768, 196

Schneider A., Smith R. E., Macciò A. V., Moore B., 2012, MNRAS, 424, 684

Schneider A., Smith R. E., Reed D., 2013, MNRAS, 433, 1573

Shi X., Fuller G. M., 1999, Physical Review Letters, 82, 2832

Springel V., 2005, MNRAS, 364, 1105

Springel V., Yoshida N., White S. D. M., 2001a, Nature, 6, 79

Springel V., White S. D. M., Tormen G., Kauffmann G., 2001b, MNRAS, 328,726

Springel V., et al., 2008, MNRAS, 391, 1685

Tegmark M., et al., 2004, Phys. Rev. D, 69, 103501 
Vegetti S., Koopmans L. V. E., 2009, MNRAS, 400, 1583

Viel M., Lesgourgues J., Haehnelt M. G., Matarrese S., Riotto A., 2005, Phys. Rev. D, 71, 063534

Viel M., Becker G. D., Bolton J. S., Haehnelt M. G., 2013, Phys. Rev. D, 88, 043502

Wang J., White S. D. M., 2007, MNRAS, 380, 93

Wang J., Frenk C. S., Navarro J. F., Gao L., Sawala T., 2012, MNRAS, 424, 2715

Weinberg D. H., Colombi S., Davé R., Katz N., 2008, ApJ, 678, 6

Wyder T. K., et al., 2005, ApJ, 619, L15

Yang L. F., Neyrinck M. C., Aragón-Calvo M. A., Falck B., Silk J., 2015, MNRAS, 451, 3606

Zehavi I., et al., 2002, ApJ, 571, 172

Zheng Z., et al., 2005, ApJ, 633, 791 